\title{
TRANSPORT OF POLLUTANT IN SHALLOW WATER A TWO TIME STEPS KINETIC METHOD
}

\author{
Emmanuel Audusse ${ }^{1}$ and Marie-Odile Bristeau ${ }^{1}$
}

\begin{abstract}
The aim of this paper is to present a finite volume kinetic method to compute the transport of a passive pollutant by a flow modeled by the shallow water equations using a new time discretization that allows large time steps for the pollutant computation. For the hydrodynamic part the kinetic solver ensures - even in the case of a non flat bottom - the preservation of the steady state of a lake at rest, the non-negativity of the water height and the existence of an entropy inequality. On an other hand the transport computation ensures the conservation of pollutant mass, a non-negativity property and a maximum principle for the concentration of pollutant and the preservation of discrete steady states associated with the lake at rest equilibrium. The interest of the developed method is to preserve these theoretical properties with a scheme that allows to disconnect the hydrodynamic time step - related to a classical CFL condition - and the transport one - related to a new CFL condition - and further the hydrodynamic calculation and the transport one. The CPU time is very reduced and we can easily solve different transport problems with the same hydrodynamic solution without large storage. Moreover the numerical results exhibit a better accuracy than with a classical method especially when using $1 \mathrm{D}$ or $2 \mathrm{D}$ regular grids.
\end{abstract}

Mathematics Subject Classification. 65M06, 76M12, 76M28, 76R05.

Received: August 23, 2002. Revised: January 21, 2003.

\section{IntRoduction}

The shallow water equations are an usual model to describe the flows in rivers or coastal areas. The conservative form is written as a first order hyperbolic system with source terms coming from the bottom topography or the friction on the bed river. Research on solution methods for these equations has received considerable attention in the past two decades and a great number of finite-volume schemes have been developed - refer to [8] or [12] for a detailed presentation. The finite volume method can be used on general triangular grids with a finite element data structure and preserves the conservativity property of the equations. It requires to compute the fluxes at the control volume interface and its stability requires some upwinding in the interpolation of the fluxes and a CFL condition on time steps - refer to [5] for a survey of its properties.

A well-known difficulty of the Saint-Venant system is the preservation of nontrivial equilibria due to the presence of the source terms. In the last years many authors treated this question along with an early idea of

Keywords and phrases. Shallow water equations, Saint-Venant system, finite volume method, kinetic scheme, transport of pollutant, time discretization.

1 Projet M3N, INRIA, Domaine de Voluceau, 78153 Le Chesnay, France.

e-mail: Emmanuel.Audusse@inria.fr, Marie-Odile.Bristeau@inria.fr 
Roe [17] to upwind the source terms at the interfaces - see for instance $[2,6,9,11]$ or [13]. On an other hand the non-negativity of the water height, especially when applications with dry areas are considered, is still a problem for several schemes.

Here we use a kinetic scheme initiated in [1] and developed in [16] and [3]. This scheme is based on a kinetic theory exposed in [15] that allows to link the shallow water equations to a kinetic equation at the mesoscopic level. In [1] only the homogeneous Saint-Venant system has a kinetic interpretation and the source terms are upwind at the macroscopic level. In [16] the source terms are also included in the kinetic formulation. The deduced schemes are conservative and we can prove analytically the non-negativity of the water height and the conservation of the equilibrium for the steady state of a lake at rest. Moreover with the second scheme the numerical solutions satisfy a discrete entropy inequality. For proofs and numerical examples, see the papers cited above.

More specifically in this paper we are concerned with addressing several questions related to the advection of a pollutant in the Saint-Venant system. It is introduced with a classical transport equation on the concentration of pollutant. The pollution phenomena have today a very large audience and many industrial applications see for instance [10] for the French electricity group EDF. Then several studies were initiated to understand better the chemical and biological background or the physical mechanisms - see for instance the study about the river Seine near Paris in [14]. But we do not find many works about the well adapted mathematical and numerical treatments. However it is an important point because the transport equation presents many properties which would be interesting to satisfy at the discrete level. Indeed as for the water height we must ensure the non-negativity of the concentration of pollutant but also a maximum principle while keeping the conservation property for the pollutant. On an other hand as we do not introduce diffusion in the model we want also to preserve the steady state equilibria related to a lake at rest. Finally we use in our scheme the fact that the characteristic velocities of the hydrodynamic and transport phenomena can be very different in order to disconnect the two time discretizations. It allows a larger time step for pollutant transport based upon the CFL condition

$$
|u| \Delta t \leq \Delta x
$$

which does not take into account the speed of sound by opposition to the hydrodynamic CFL. It is particularly useful when numerous pollutants are computed or when different pollutant problems are computed with the same hydrodynamic background as it appears often in water quality questions.

The outline of this paper is the following. After presenting the equations in Section 2, we briefly recall the kinetic formulation, the deduced scheme and its extension to the advection equation in Section 3. Then we prove in Section 4 the non-negativity properties, the maximum principle for the pollutant and the preservation of equilibria. Finally in Section 5 we present a two time steps scheme and the associated time discretization. In Section 6 we present some numerical results to illustrate the improvements of the method. Then in Section 7 we discuss an extension to the two dimensional case and we present some numerical results on realistic geometries.

\section{EQUATIONS}

The one dimensional shallow water system allows to describe the flow in an ideal rectangular river, at time $t \in \mathbb{R}_{+}$and at the point $x \in \mathbb{R}$, through the water height $h(t, x) \in \mathbb{R}_{+}$and the velocity $u(t, x) \in \mathbb{R}$, by the hyperbolic system

$$
\begin{array}{r}
\frac{\partial h}{\partial t}+\frac{\partial(h u)}{\partial x}=S(t, x) \\
\frac{\partial(h u)}{\partial t}+\frac{\partial}{\partial x}\left(h u^{2}+\frac{g h^{2}}{2}\right)+g h \frac{\partial z}{\partial x}=0
\end{array}
$$


with $g$ the gravity acceleration and $z(x) \in \mathbb{R}$ the bottom topography and where $S(t, x) \in \mathbb{R}$ denotes the sources of water (in $m \times s^{-1}$ ). Therefore $h+z$ is the level of water surface and in the following we denote $q=h u$ the discharge.

These equations were originally written by Saint-Venant in [18]. Gerbeau and Perthame present in [7] a derivation from the Navier-Stokes system. The system $(2.1,2.2)$ corresponds to a very simple case. Other terms can be added in the right-hand side, in order to take into account frictions on the bottom or other phenomena.

To perform the analysis of transport of pollutant in this ideal river, we add a third equation

$$
\frac{\partial(h T)}{\partial t}+\frac{\partial(h u T)}{\partial x}=T_{S} S
$$

where $T(t, x) \in \mathbb{R}_{+}$is the concentration of pollutant and $T_{S}$ are the given values of the concentration of pollutant at the sources $S$. In the following we denote $e=h T$ the quantity of pollutant in the flow. We can also write this equation on the nonconservative form where 'monotonicity' is better seen

$$
\frac{\partial T}{\partial t}+u \frac{\partial T}{\partial x}=\frac{\left(T_{S}-T\right) S}{h}
$$

It is a classical transport equation. Here we suppose that the pollutant is passive and does not interact with the flow. But in some cases, other phenomena like sedimentation, erosion, birth or death of particles have to be considered.

We can write this system in the conservative and compact form

$$
\frac{\partial U}{\partial t}+\frac{\partial F(U)}{\partial x}=B(U)
$$

with

$$
U=\left(\begin{array}{c}
h \\
q \\
e
\end{array}\right), \quad F(U)=\left(\begin{array}{c}
q \\
\frac{q^{2}}{h}+\frac{g h^{2}}{2} \\
\frac{q e}{h}
\end{array}\right), B(U)=\left(\begin{array}{c}
S \\
-g h \frac{\partial z}{\partial x} \\
T_{S} S
\end{array}\right)
$$

\section{The KINETIC SCHEME}

\subsection{Kinetic interpretation of the shallow water equations}

We introduce here a kinetic approach to system (2.1)-(2.3) written with $S=0$. Then we can deduce from the discretization of the kinetic equation a "kinetic scheme" for the macroscopic system.

Let $\chi(w)$ be an even and compactly supported probability defined on $\mathbb{R}$ satisfying that its second moment is equal to one. Then we introduce two microscopic densities of particles $M(t, x, \xi)$ and $N(t, x, \xi)$ defined by a Gibbs equilibrium

$$
\begin{aligned}
& M(t, x, \xi)=\frac{h(t, x)}{c(t, x)} \chi\left(\frac{\xi-u(t, x)}{c(t, x)}\right), \\
& N(t, x, \xi)=\frac{e(t, x)}{c(t, x)} \chi\left(\frac{\xi-u(t, x)}{c(t, x)}\right),
\end{aligned}
$$

with

$$
c(t, x)^{2}=\frac{g h(t, x)}{2}
$$


We denote

$$
G(t, x, \xi)=\left(\begin{array}{c}
M(t, x, \xi) \\
N(t, x, \xi)
\end{array}\right) .
$$

Theorem 3.1. The functions $(h, q, e)(t, x)$ are strong solutions of the shallow water system (2.1)-(2.3) if and only if $G(t, x, \xi)$ is solution of the kinetic equation

$$
\frac{\partial G}{\partial t}+\xi \frac{\partial G}{\partial x}-g \frac{\partial z}{\partial x} \frac{\partial G}{\partial \xi}=Q(t, x, \xi)
$$

where $Q(t, x, \xi)$ is a "collision term" equal to zero at the macroscopic level.

Proof. The result is obtained by a simple integration in $\xi$ of the equation (3.4) against the matrix $K(\xi)$

$$
K(\xi)=\left(\begin{array}{ll}
1 & 0 \\
\xi & 0 \\
0 & 1
\end{array}\right) .
$$

The non-linear Saint-Venant system is now reduced to a linear transport system on nonlinear quantities $M$ and $N$, for which it is easier to find a simple numerical scheme with good theoretical properties. For a detailed proof of the hydrodynamic part of the kinetic interpretation refer to [1] and for the treatment of the source term at this microscopic level, see [16].

\subsection{The kinetic scheme}

We now describe the kinetic scheme without taking into account the source term due to the bottom topography. Indeed we are more interested here with the treatment of the pollutant and for the simplicity of the purpose we restrict ourselves to the flat bottom case. For a complete hydrodynamic presentation we refer to [1] or $[16]$.

To approximate the solution $U(t, x), x \in X \in \mathbb{R}, t \geq 0$, of the shallow water equations with transport of pollutant by discrete values $U_{i}^{n}, i \in I \subset \mathbb{Z}, n \in \mathbb{N}$ we consider as usual a grid of points $x_{i+1 / 2}, i \in I$,

$$
\cdots<x_{i-1 / 2}<x_{i+1 / 2}<x_{i+3 / 2}<\ldots,
$$

and we define the cells (or finite volumes) and their lengths

$$
\left.C_{i}=\right] x_{i-1 / 2}, x_{i+1 / 2}\left[, \quad \Delta x_{i}=x_{i+1 / 2}-x_{i-1 / 2}>0 .\right.
$$

We shall denote also $x_{i}=\left(x_{i-1 / 2}+x_{i+1 / 2}\right) / 2$ and we consider a time-step $\Delta t^{n}>0$ - that will be specified later - and define the discrete times by

$$
t^{n}=\sum_{0}^{n-1} \Delta t^{k}, \quad n \in \mathbb{N}^{*} .
$$

Now being given a piecewise constant approximation of the initial data, we must find a formula of the form

$$
U_{i}^{n+1}=U_{i}^{n}+\sigma_{i}^{n}\left(F_{i-\frac{1}{2}}^{n}-F_{i+\frac{1}{2}}^{n}\right),
$$

where

$$
U_{i}^{n}=\left(\begin{array}{c}
h_{i}^{n} \\
q_{i}^{n} \\
e_{i}^{n}
\end{array}\right)
$$


and $U_{i}^{n+1}$ are the piecewise constant approximations at times $t^{n}$ and $t^{n+1}$ on the cell $C_{i}$, where $\sigma_{i}^{n}$ is defined by

$$
\sigma_{i}^{n}=\frac{\Delta t^{n}}{\Delta x_{i}}
$$

and where the fluxes $F_{i-\frac{1}{2}}^{n}$ and $F_{i+\frac{1}{2}}^{n}$ must be specified.

Here we use the kinetic interpretation to precise the formula (3.5). First we define two discrete densities of particles $M_{i}^{n}(\xi)$ and $N_{i}^{n}(\xi)$ by

$$
\begin{aligned}
M_{i}^{n}(\xi) & =\frac{h_{i}^{n}}{c_{i}^{n}} \chi\left(\frac{\xi-u_{i}^{n}}{c_{i}^{n}}\right), \\
N_{i}^{n}(\xi) & =\frac{e_{i}^{n}}{c_{i}^{n}} \chi\left(\frac{\xi-u_{i}^{n}}{c_{i}^{n}}\right),
\end{aligned}
$$

and the corresponding quantity $G_{i}^{n}(\xi)$. Then we define a new density function $G_{i}^{n+1}(\xi)$ at time $t^{n+1}$ with applying a simple upwind scheme for the discrete version of the kinetic equation (3.4) for every $\xi$ and without taking account the right-hand side

$$
G_{i}^{n+1}(\xi)=G_{i}^{n}(\xi)-\xi \sigma_{i}^{n}\left(G_{i+\frac{1}{2}}^{n}(\xi)-G_{i-\frac{1}{2}}^{n}(\xi)\right)
$$

with

$$
G_{i+\frac{1}{2}}^{n}(\xi)=\left\{\begin{array}{lll}
G_{i}^{n}(\xi) & \text { if } \quad \xi \geq 0 \\
G_{i+1}^{n}(\xi) & \text { if } \quad \xi<0
\end{array}\right.
$$

This new density function is not an equilibrium but thanks to the property of the right-hand side of (3.4) we can recover the macroscopic quantity at time $t^{n+1}$ by a simple integration in $\xi$. Finally we can precise the macroscopic formula (3.5) with

$$
F_{i+\frac{1}{2}}^{n}=F\left(U_{i}^{n}, U_{i+1}^{n}\right)=F^{+}\left(U_{i}^{n}\right)+F^{-}\left(U_{i+1}^{n}\right),
$$

where

$$
\begin{aligned}
F^{-}\left(U_{i}^{n}\right) & =\int_{\xi \in \mathbb{R}_{-}} \xi K(\xi) G_{i}^{n}(\xi) \mathrm{d} \xi \\
F^{+}\left(U_{i}^{n}\right) & =\int_{\xi \in \mathbb{R}_{+}} \xi K(\xi) G_{i}^{n}(\xi) \mathrm{d} \xi .
\end{aligned}
$$

A detailed expression of $F^{+}\left(U_{i}\right)$ can also be written

$$
F^{+}\left(U_{i}\right)=\left(\begin{array}{c}
F_{h}^{+}\left(U_{i}\right) \\
F_{q}^{+}\left(U_{i}\right) \\
F_{e}^{+}\left(U_{i}\right)
\end{array}\right)=h_{i} \int_{w \geq \frac{-u_{i}}{c_{i}}}\left(u_{i}+w c_{i}\right)\left(\begin{array}{c}
1 \\
u_{i}+w c_{i} \\
T_{i}
\end{array}\right) \chi(w) \mathrm{d} w .
$$

This kinetic method is interesting because it gives a very simple and natural way to propose a numerical flux through the kinetic interpretation and, if we can perform analytically the integration in (3.9), it is numerically powerful because the kinetic level disappears and the scheme is written directly as a macroscopic scheme.

Notice also that according to the above derivation, the pollutant flux $F_{e}^{+}\left(U_{i}\right)$ that we refer to as the classical kinetic scheme and that we denote $F_{k, e}^{+}\left(U_{i}\right)$ in the following, has a very simple expression

$$
F_{k, e}^{ \pm}\left(U_{i}\right)=T_{i} F_{h}^{ \pm}\left(U_{i}\right) .
$$

Nevertheless we will not use it in practice because of a lack of accuracy as we will show in the next section. 


\subsection{Preservation of the equilibria}

We do not treat here the problem of the hydrodynamic equilibria because in the case of a flat bottom they are trivially preserved. We refer to [1] or [16] where the authors prove analytically the preservation of the lake at rest equilibrium whatever is the bottom topography.

We are now interested with the problem of the conservation of equilibria for the pollutant. Consider the situation of a lake at rest - with a flat bottom or not - and a numerical scheme that preserves it. Then the numerical discharge vanishes and the water height is constant. So

$$
F_{h}\left(U_{j}, U_{j+1}\right)=0 \quad \forall j \in \mathbb{Z}
$$

and so due to $(3.6)$

$$
F_{h}^{+}\left(U_{j}\right)=-F_{h}^{-}\left(U_{j+1}\right) \quad \forall j \in \mathbb{Z},
$$

but - from (3.9) - these two fluxes are not equal to zero.

Now put some pollutant with concentration equal to one at the node $\mathrm{i}$ of the mesh and zero everywhere else. As there is no diffusion in the model this situation is an equilibrium. But numerically - from (3.10) - the classical kinetic scheme induces

$$
\begin{aligned}
& F_{k, e}^{ \pm}\left(U_{i}\right) \neq 0, \\
& F_{k, e}^{ \pm}\left(U_{j}\right)=0 \quad \forall j \neq i,
\end{aligned}
$$

and then (3.6) shows immediately that the equilibrium is not preserved for the concentration of pollutant. For long time integration the classical kinetic scheme will create very large diffusion for the pollutant and thus we discard it.

We rather introduce some upwinding in the transport advection depending on the sign of the total mass flux. Then the pollutant flux vanishes with the total mass flux. It is done with the introduction of the new pollutant flux $F_{u k, e}\left(U_{i}, U_{i+1}\right)$ defined in the following formula that replaces (3.10)

$$
F_{u k, e}\left(U_{i}, U_{i+1}\right)=T_{i+\frac{1}{2}} F_{h}\left(U_{i}, U_{i+1}\right)
$$

where

$$
T_{i+\frac{1}{2}}=\left(\begin{array}{ccc}
T_{i} & \text { for } & F_{h} \geq 0 \\
T_{i+1} & \text { for } & F_{h} \leq 0
\end{array}\right)
$$

In the following we call this new scheme the upwind kinetic scheme.

Theorem 3.2. With the pollutant flux (3.12, 3.13) the upwind kinetic scheme (3.5)-(3.8) preserves the pollutant equilibrium in a lake at rest.

Proof. The hydrodynamic computation is unchanged and so (3.11) is always true. Then from (3.12, 3.13) we have immediately

$$
F_{u k, e}\left(U_{j}, U_{j+1}\right)=0 \quad \forall j \in \mathbb{Z} .
$$

\section{Properties of the scheme}

\subsection{Positivity of the water height}

In addition to the preservation of the hydrodynamic and transport equilibria in a lake at rest the upwind kinetic scheme has numerous good properties. As we are interested here with the coupling between the shallow water system and a transport equation, the problem of the source term is not treated. As for the hydrodynamic part of the proofs we restrict ourselves to the case of a flat bottom. But notice that these results also hold true 
with a non-flat bottom, $c f$. [1] or [16] for complete proofs on the hydrodynamic part. In Sections 6 and 7 some numerical examples with a non-flat bottom are presented using our scheme.

Theorem 4.1. The scheme is consistent and conservative. It ensures the non-negativity of the water height under the CFL condition

$$
\Delta t^{n} \leq \min \left(\frac{\Delta x_{i}}{\left|u_{i}^{n}\right|+w_{M} c_{i}^{n}}\right)
$$

where $2 w_{M}$ is the size of the compact support of $\chi$.

Proof. Consistency and conservativity are easily deduced by (3.5)-(3.8).

To prove the positivity of the water height let us go back to the microscopic level. Suppose we have $h_{i}^{n} \geq$ $0 \forall i \in \mathbb{Z}$. From the definition of the function $M$ in (3.1) and the positivity of the function $\chi$, we immediately deduce

$$
M_{i}^{n}(\xi) \geq 0 \quad \forall i .
$$

We now introduce the quantities $\xi_{+}, \xi_{-}$defined by

$$
\xi_{+}=\max (0, \xi), \quad \xi_{-}=\max (0,-\xi),
$$

and so we write the upwind microscopic scheme deduced by (3.4) in the form

$$
f_{i}^{n+1}(\xi)=\left(1-\sigma_{i}^{n}|\xi|\right) M_{i}^{n}(\xi)+\sigma_{i-1}^{n} \xi_{+} M_{i-1}^{n}(\xi)+\sigma_{i+1}^{n} \xi_{-} M_{i+1}^{n}(\xi) .
$$

Then, for each $j$, either the value of $\xi$ is such that

$$
\left|\xi-u_{j}^{n}\right| \geq w_{M} c_{j}^{n},
$$

and then from the definitions of $w_{M}$ and of function $M$ we have

$$
M_{j}^{n}(\xi)=0,
$$

or the value of $\xi$ is such that

$$
\left|\xi-u_{j}^{n}\right| \leq w_{M} c_{j}^{n},
$$

which implies that $|\xi| \leq\left(\left|u_{j}^{n}\right|+w_{M} c_{j}^{n}\right)$ and then, using the CFL condition (4.1), we obtain

$$
\sigma_{j}^{n}|\xi| \leq 1 .
$$

Therefore in the relation $(4.3), f_{i}^{n+1}(\xi)$ is a convex combination of nonnegative quantities and thus

$$
f_{i}^{n+1}(\xi) \geq 0 .
$$

With a simple integration in $\xi$, we obtain

$$
h_{i}^{n+1} \geq 0
$$

\subsection{Positivity of the concentration of pollutant}

Theorem 4.2. The upwind kinetic scheme (3.5)-(3.8) and (3.12, 3.13) preserves the positivity of the concentration of pollutant. 
Proof. We present first another equivalent formula for the pollutant flux $(3.12,3.13)$

$$
F_{u k, e}\left(U_{i}, U_{i+1}\right)=T_{i} F_{h}\left(U_{i}, U_{i+1}\right)_{+}-T_{i+1} F_{h}\left(U_{i}, U_{i+1}\right)_{-},
$$

where we refer to (4.2) for the definitions of the positive and negative parts.

We assume that the concentration of pollutant is nonnegative at time $t^{n}$ and we drop the superscripts $\mathrm{n}$ for simplicity. Then

$$
(h T)_{i}^{n+1}=(h T)_{i}+\sigma_{i}\left(F_{u k, e}\left(U_{i-1}, U_{i}\right)-F_{u k, e}\left(U_{i}, U_{i+1}\right)\right),
$$

that is exactly with (4.4)

$$
\begin{aligned}
(h T)_{i}^{n+1}= & (h T)_{i} \\
& +\sigma_{i}\left[T_{i-1} F_{h}\left(U_{i-1}, U_{i}\right)_{+}-T_{i} F_{h}\left(U_{i-1}, U_{i}\right)_{-}\right. \\
& \left.-T_{i} F_{h}\left(U_{i}, U_{i+1}\right)_{+}+T_{i+1} F_{h}\left(U_{i}, U_{i+1}\right)_{-}\right] .
\end{aligned}
$$

Thanks to the non-negativity of the concentration of pollutant at time $t^{n}$ we can write the inequality

$$
(h T)_{i}^{n+1} \geq T_{i}\left(h_{i}-\sigma_{i}\left(F_{h}\left(U_{i-1}, U_{i}\right)_{-}+F_{h}\left(U_{i}, U_{i+1}\right)_{+}\right)\right) .
$$

Now we remark from $(3.7,3.8)$ and due to the positivity of function $M$ that

$$
\begin{aligned}
& F^{-}\left(U_{j}\right) \leq 0 \quad \forall j \in \mathbb{Z}, \\
& F^{+}\left(U_{j}\right) \geq 0 \quad \forall j \in \mathbb{Z},
\end{aligned}
$$

and so we can write, using (3.6),

$$
(h T)_{i}^{n+1} \geq T_{i}\left(h_{i}-\sigma_{i}\left(F_{h}^{+}\left(U_{i}\right)-F_{h}^{-}\left(U_{i}\right)\right)\right) .
$$

Until now we have not used the fact that we are on a flat bottom and if we forget the concentrations of the pollutant in the precedent expression we recognize a step of the proof of the non-negativity of the water height. We can conclude the two proofs are linked and so if the scheme ensures the non-negativity of the water height then it ensures automatically the non-negativity of the concentration of pollutant - whatever is the bottom topography. More precisely in the case of a flat bottom the end of the proof is to use the detailed expression of the fluxes to minimize the right-hand side. Indeed from (3.9) and thanks to the property of $\chi$

$$
\begin{aligned}
F_{h}^{+}\left(U_{i}\right)-F_{h}^{-}\left(U_{i}\right)= & h_{i} \int_{w \geq \frac{-u_{i}}{c_{i}}}\left(u_{i}+w c_{i}\right) \chi(w) \mathrm{d} w \\
& -h_{i} \int_{w \leq \frac{-u_{i}}{c_{i}}}\left(u_{i}+w c_{i}\right) \chi(w) \mathrm{d} w \\
\leq & h_{i} \int_{w \in \mathbb{R}}\left(u_{i}+|w| c_{i}\right) \chi(w) \mathrm{d} w \\
\leq & h_{i}\left(u_{i}+w_{M} c_{i}\right) .
\end{aligned}
$$

Therefore, we deduce

$$
(h T)_{i}^{n+1} \geq T_{i} h_{i}\left(1-\sigma_{i}\left(u_{i}+w_{M} c_{i}\right)\right)
$$

and the CFL condition (4.1) ensures also the non-negativity of concentration of pollutant. 


\subsection{Maximum principle for the concentration of pollutant}

Theorem 4.3. The upwind kinetic scheme (3.5)-(3.8) and (3.12, 3.13) ensures a maximum principle for the concentration of pollutant. Indeed it satisfies

$$
\forall n \quad \forall i \quad T_{i}^{n+1} \leq \max \left(T_{i-1}^{n}, T_{i}^{n}, T_{i+1}^{n}\right) .
$$

Proof. We rewrite (4.5) in another form - still dropping the superscripts $\mathrm{n}$ for more readability

$$
\begin{aligned}
(h T)_{i}^{n+1}= & T_{i}\left(h_{i}-\sigma_{i}\left(F_{h}\left(U_{i-1}, U_{i}\right)_{-}+F_{h}\left(U_{i}, U_{i+1}\right)_{+}\right)\right) \\
& +T_{i-1} \sigma_{i} F_{h}\left(U_{i-1}, U_{i}\right)_{+}+T_{i+1} \sigma_{i} F_{h}\left(U_{i}, U_{i+1}\right)_{-} .
\end{aligned}
$$

The two last parts of the right-hand side are clearly nonnegative and we established in the precedent proof it is true for the first part too. So we can maximize the left quantity

$$
\begin{aligned}
(h T)_{i}^{n+1} \leq & \max \left(T_{i-1}, T_{i}, T_{i+1}\right)\left(h_{i}+\sigma_{i}\left(-F_{h}\left(U_{i-1}, U_{i}\right)_{-}-F_{h}\left(U_{i}, U_{i+1}\right)_{+}\right.\right. \\
& \left.\left.+F_{h}\left(U_{i-1}, U_{i}\right)_{+}+F_{h}\left(U_{i}, U_{i+1}\right)_{-}\right)\right) .
\end{aligned}
$$

A simple reorganization of the right-hand side gives with the formulae (3.5) and (3.6)

$$
(h T)_{i}^{n+1} \leq h_{i}^{n+1} \max \left(T_{i-1}, T_{i}, T_{i+1}\right) .
$$

\section{LARGER TIME STEPS FOR THE POLLUTANT}

\subsection{Motivation}

It is well known that the two eigenvalues of the Saint-Venant system are related to the velocity of the flow and to the water height by the formulae $u+\sqrt{g h}$ and $u-\sqrt{g h}$. The eigenvalue of the transport equation is simply equal to the velocity of the flow. So it appears in the cases where the Froude number defined by

$$
F r=\frac{u}{\sqrt{g h}}
$$

is small - which is almost always the case for a classical river - that the characteristic time for information transfer is very different for the hydrodynamic and for the transport parts. Then if the CFL number is relevant for the hydrodynamic computation it is not connected to the physical background of the transport phenomenon. Therefore it could be interesting to create an adaptive numerical scheme that allows to disconnect the two time discretizations. Especially in some realistic applications, when we can have to treat twenty or thirty different pollutants - and some of them are modeled by more complicated equations than $(2.3)$ - or when we want to test different phenomena of pollution in the same hydrodynamic background. So we would like to solve only the relevant transport states and to store only the useful global hydrodynamic informations while ensuring all the properties presented in the previous section.

The way to reach our goal is to disconnect the transport solution from the CFL condition (4.1). Indeed this time step condition is strictly related to the eigenvalues of the hydrodynamic process but is not necessary to ensure the transport properties. In fact it appears that the only theoretical time step restriction is given by the positivity of the right-hand side of (4.6) - the non-negativity property that we can then deduce is also sufficient to prove the maximum principle. It is clear that the CFL condition (4.1) is more restrictive. On an other hand notice that the positivity of the right-hand side of (4.6) is the less restrictive formula that we can obtain, if we impose that the non-negativity condition for the concentration of pollutant depends only of the hydrodynamic process. 
At last notice that the new condition we introduce exhibits a term which is analogous to a velocity and so it is in accordance with the remark on the eigenvalues that we make first. Furthermore in the very simple case where we consider a stationary flow on a flat bottom - constant discharge and water height - the non-negativity of the right-hand side of (4.6) is reduced to

$$
\Delta t^{n} \leq \min \left(\frac{\Delta x_{i}}{\left|u_{i}^{n}\right|}\right)
$$

\subsection{Algorithm}

We now consider two different time step conditions. Notice that the CFL condition (4.1) is an a priori condition - we need only to know the hydrodynamic state at time $t^{n}$ to compute the time step - while the transport time step condition (4.6) is an a posteriori condition - we must compute the mass fluxes before we compute the time step condition. On an other hand it is convenient to manage with the hydrodynamic and the pollutant quantities at the same time and so the transport time step must be the sum of some hydrodynamic time steps.

So we propose the following algorithm: starting from a given state we perform the hydrodynamic computation - time step and fluxes - then we compute the transport time step condition (4.6) with this time step and these fluxes. If (4.6) is satisfied we perform another hydrodynamic computation, we sum the hydrodynamic time steps and fluxes and we compute (4.6) with these sums. We continue this algorithm until (4.6) is not satisfied. Then we compute the new pollutant state.

Before we present precisely the algorithm let us make some useful remarks. First the hydrodynamic computation is exactly the same as in the case of the upwind solution. It means that our strategy preserves the fact that the pollutant has no influence on the hydrodynamic phenomena and it ensures that the positivity property is preserved. Second the transport time step is computed as a sum of hydrodynamic time steps. It means that we can not know a priori the transport time step but it allows us to be very adaptive and the transport time step to be as large as possible in relation to the hydrodynamic conditions. Third the reference to the transport time step condition ensures the preservation of the properties of the precedent section.

Let us now present the details of the algorithm:

\section{Initialization}

- Start from the state $h_{i}^{n}, q_{i}^{n}, e_{i}^{n}$.

- Initialization of the transport time step and total fluxes:

$$
\begin{aligned}
\Delta t^{n} & =0, \\
G_{h}^{n}\left(U_{i-1}, U_{i}\right) & =0, \\
G_{h}^{n}\left(U_{i}, U_{i+1}\right) & =0 .
\end{aligned}
$$

- Initialization of the step indicator:

$$
k=0 .
$$

- Initialization of the small time step hydrodynamic computation:

$$
\begin{gathered}
h_{i}^{n, 0}=h_{i}^{n}, \\
q_{i}^{n, 0}=q_{i}^{n} .
\end{gathered}
$$

\section{Computation at the step $n, k$}

(1) Computation of hydrodynamic time step $\Delta t^{n, k}$ deduced by the CFL condition (4.1) related to $h_{i}^{n, k}$ and $Q_{i}^{n, k}$.

(2) Computation of the partial fluxes $F^{+}\left(U_{i}\right)^{n, k}$ and $F^{-}\left(U_{i}\right)^{n, k}$ with the formula (3.9). 
(3) Computation of the total interfaces fluxes $F^{n, k}\left(U_{i}, U_{i+1}\right)$ and $F^{n, k}\left(U_{i-1}, U_{i}\right)$ with the formula (3.6).

(4) Test based on an extension of the non-negativity condition (4.6):

$$
h_{i}^{n}-\frac{1}{\Delta x_{i}}\left(\sum_{0}^{k} \Delta t^{n, j} F_{h}^{n, j}\left(U_{i}, U_{i+1}\right)\right)_{+}-\frac{1}{\Delta x_{i}}\left(\sum_{0}^{k} \Delta t^{n, j} F_{h}^{n, j}\left(U_{i-1}, U_{i}\right)\right)_{-} \geq 0
$$

\section{Updating of the solution}

- If (5.1) is true then update the hydrodynamic quantities

$$
\begin{aligned}
h_{i}^{n, k+1} & =h_{i}^{n, k}-\frac{\Delta t^{n, k}}{\Delta x_{i}}\left(F_{h}^{n, k}\left(U_{i}, U_{i+1}\right)-F_{h}^{n, j}\left(U_{i-1}, U_{i}\right)\right), \\
Q_{i}^{n, k+1} & =Q_{i}^{n, k}-\frac{\Delta t^{n, k}}{\Delta x_{i}}\left(F_{Q}^{n, k}\left(U_{i}, U_{i+1}\right)-F_{Q}^{n, j}\left(U_{i-1}, U_{i}\right)\right),
\end{aligned}
$$

the transport quantities

$$
\begin{aligned}
G_{h}^{n}\left(U_{i-1}, U_{i}\right) & =G_{h}^{n}\left(U_{i-1}, U_{i}\right)+\Delta t^{n, k} F_{h}^{n, j}\left(U_{i-1}, U_{i}\right), \\
G_{h}^{n}\left(U_{i}, U_{i+1}\right) & =G_{h}^{n}\left(U_{i}, U_{i+1}\right)+\Delta t^{n, k} F_{h}^{n, k}\left(U_{i}, U_{i+1}\right), \\
\Delta t^{n} & =\Delta t^{n}+\Delta t^{n, k},
\end{aligned}
$$

and the step indicator

$$
k=k+1 .
$$

Then go to the Computation step.

- If (5.1) is false then update the hydrodynamic quantities

$$
\begin{aligned}
h_{i}^{n+1} & =h_{i}^{n, k}, \\
Q_{i}^{n+1} & =Q_{i}^{n, k},
\end{aligned}
$$

and the new concentration of pollutant

$$
\begin{aligned}
(h T)_{i}^{n+1}= & (h T)_{i}^{n} \\
& +\frac{1}{\Delta x_{i}}\left(T_{i-1}\left(G_{h}^{n}\left(U_{i-1}, U_{i}\right)\right)_{+}+T_{i}\left(G_{h}^{n}\left(U_{i-1}, U_{i}\right)\right)_{-}\right) \\
& -\frac{1}{\Delta x_{i}}\left(T_{i}\left(G_{h}^{n}\left(U_{i}, U_{i+1}\right)\right)_{+}+T_{i+1}\left(G_{h}^{n}\left(U_{i}, U_{i+1}\right)\right)_{-}\right) .
\end{aligned}
$$

Then go to the Initialization step.

\subsection{Consistency, conservativity, positivity, maximum principle and preservation of equilibria}

The hydrodynamic computation is unchanged and so the scheme is still consistent and conservative for its hydrodynamic part and the non-negativity of the water height is still ensured by the CFL condition introduced at each hydrodynamic time step.

On an other hand the consistency and the conservativity are also preserved for the transport part thanks to the form of the algorithm and the non-negativity of the concentration of pollutant is ensured at each transport time step by the test (5.1). The maximum principle is satisfied too because the essential point - that is the non-negativity of each term in equation (4.8) - is also ensured by the same test (5.1). 
Finally the preservation of the different equilibria in a lake at rest is always true because even if the scheme contains now two different time discretizations its global form for hydrodynamic part on one hand and for transport part on the other hand is not modified.

\section{NumericAl RESULTS}

\subsection{Transport of pollutant in a flat bottom channel with constant discharge}

We consider a flat bottom channel and the associated stationary solution of the shallow water equations with constant discharge and water height. Taking into account this hydrodynamic background and the form of the transport equation (2.4) a polluted area will be simply transported with the constant speed $u_{\mathrm{c}}$ of the flow

$$
\frac{\partial T}{\partial t}+u_{\mathrm{c}} \frac{\partial T}{\partial x}=0
$$

The interest of this very simple case is to clearly exhibit the influence of the Froude number and the diffusion of the schemes by comparing the numerical results with an exact solution.

The data of the numerical test are

$$
\begin{aligned}
& \text { Spatial domain: } x \in[0,500] \\
& \text { Uniform mesh with } 101 \text { nodes. } \\
& \text { Water height: } 1 \mathrm{~m} . \\
& \text { Initial concentration of pollutant: } \begin{cases}1 & \text { if } x \in[20,70] \\
0 & \text { if not. }\end{cases}
\end{aligned}
$$

Then we compute the solution for different Froude numbers $F r$ - the simulation time is equal to $\frac{100}{F r}-$ and we present in Table 1 the number of necessary transport and hydrodynamic time steps. We do not compute the case of the lake at rest since we established that the hydrodynamic and transport equilibria were preserved by both schemes.

TABLE 1. Comparison between numbers of hydrodynamic and transport time steps.

\begin{tabular}{|c|c|c|c|}
\hline Froude number & Simulation Time & Transport Steps & Hydrodynamic Steps \\
\hline 10. & 10 & 71 & 71 \\
\hline 1. & 100 & 70 & 140 \\
\hline 0.1 & 1000 & 64 & 830 \\
\hline 0.01 & 10000 & 63 & 7735 \\
\hline
\end{tabular}

First of all these results exhibit the auto-adaptive aspect of the two time steps method. Indeed the ratio between the number of transport and hydrodynamic time steps appears to be a function of the Froude number. Then the results imply clearly that in the cases where the Froude number is small the computation cost economy could be very important because the gain in term of number of time steps is very large. It will authorize also to apply easily different pollution models on the same hydrodynamic background since we need to store only one global hydrodynamic information - which corresponds to the global mass flux at each interface - for each transport time step.

We are interested now with the numerical results. We just mention that the scheme preserves the hydrodynamic stationarity and we present in Figures 1 and 2 the numerical results for the pollutant concentration. We indicate the exact solution and the results for the upwind kinetic scheme and the two time steps scheme. First notice that the numerical results are in accordance with the different properties of the schemes established in the precedent sections. Then it appears that the two time steps method does not only improve the computation 

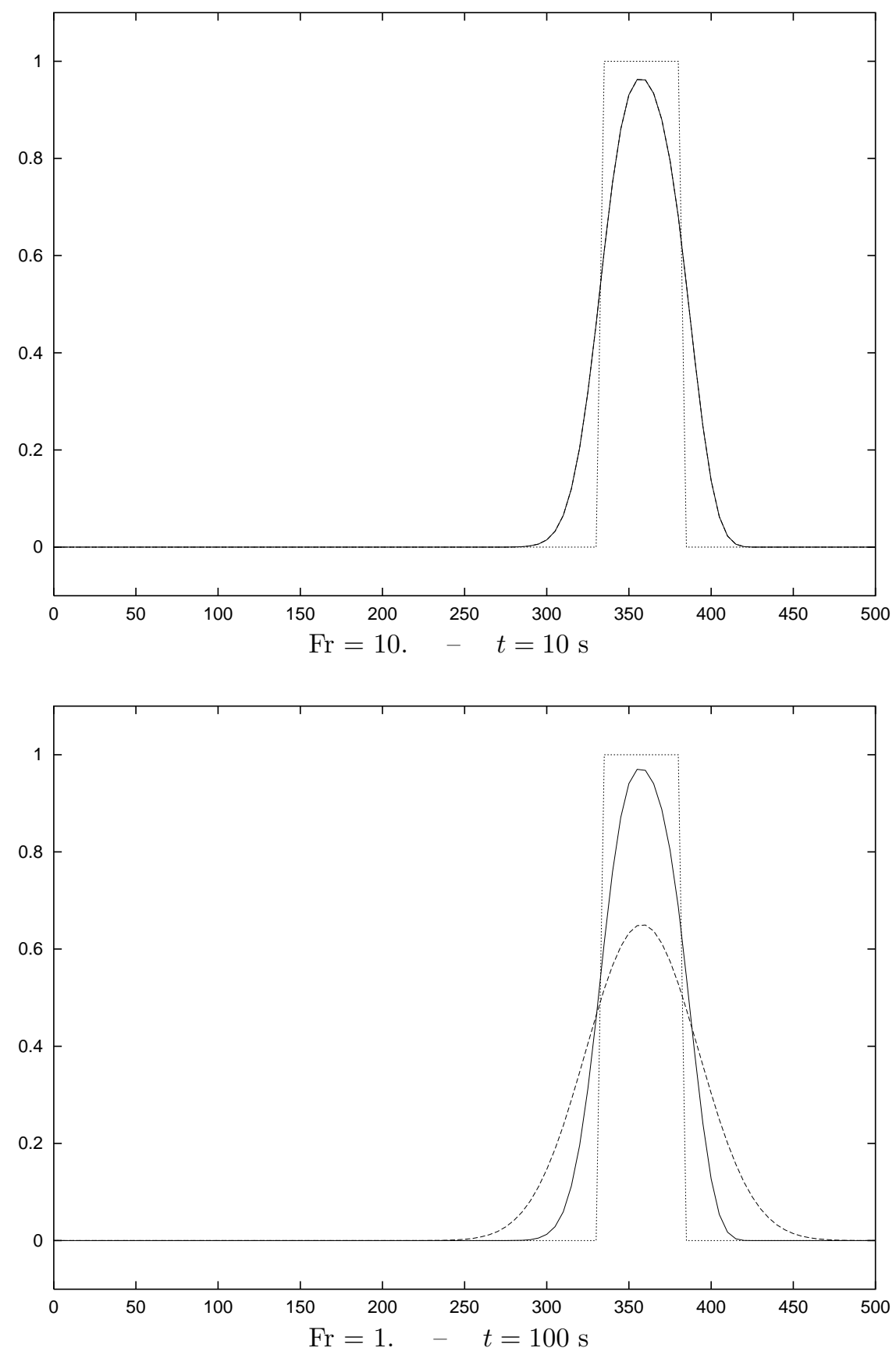

Figure 1. Concentration of pollutant for some constant discharge problems. Exact solution (dotted line); upwind scheme (dash line); two time steps scheme (continuous line).

time but also the precision of the numerical results. Indeed the two time steps solution is always closer to the exact solution than the upwind kinetic one. And lower is the Froude number larger is the difference between the two schemes: if the results are the same for the case where the Froude number is equal to 10 - we see also in Table 1 that the numbers of time steps are the same in this case - the upwind kinetic results are worse and 

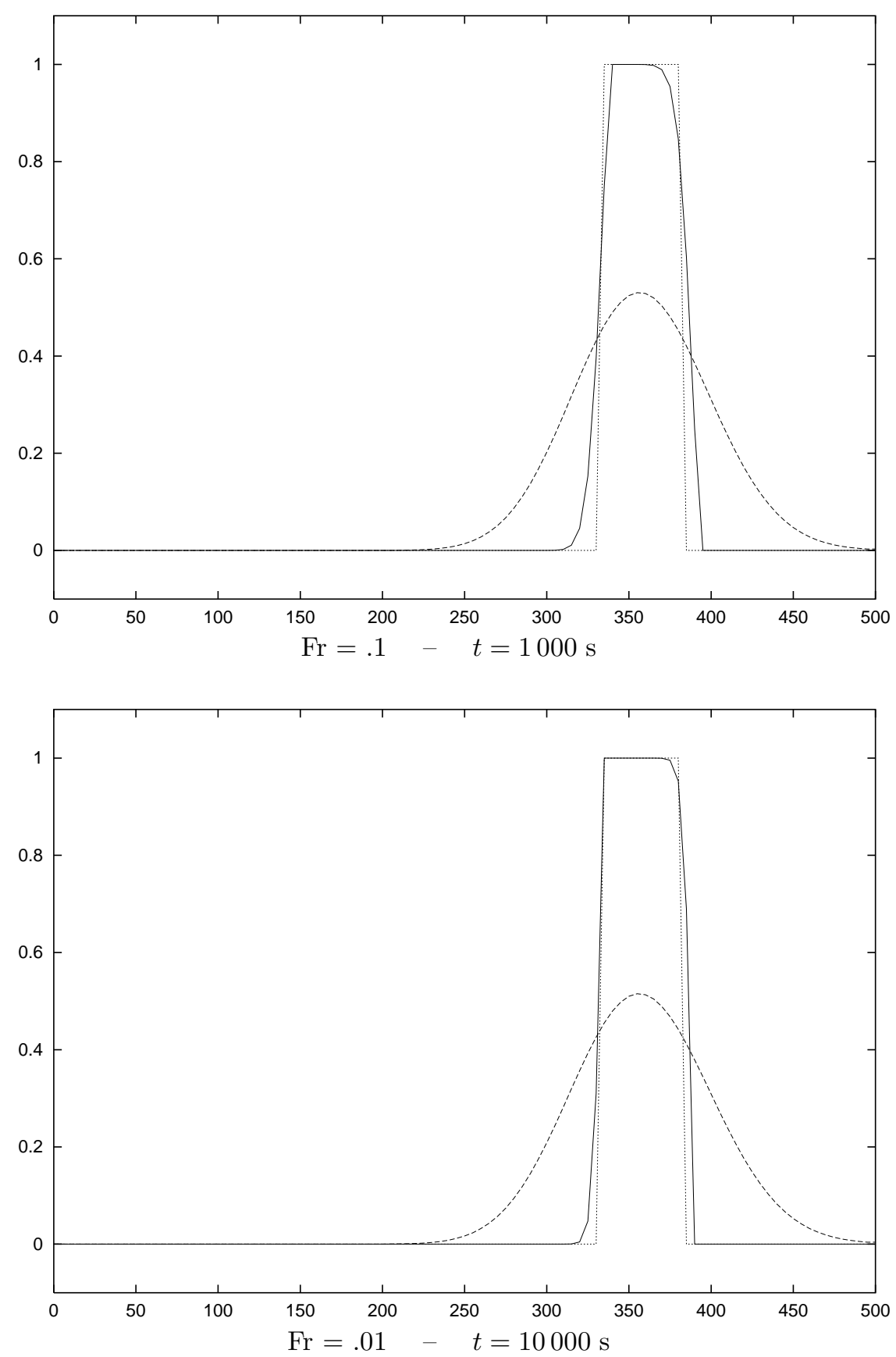

FiguRE 2. Concentration of pollutant for some constant discharge problems. Exact solution (dotted line); upwind scheme (dash line); two time steps scheme (continuous line).

worse when the Froude number decreases - because it corresponds to a large increasing of the simulation time and then of the number of hydrodynamic time steps - while the two time steps results are better and better because here the number of transport time steps is very stable. To quantify this analysis we present in Table 2 the discrete relative $L^{1}$ error on the pollutant concentration for both schemes and for the different cases. 
TABLE 2. Discrete relative $L^{1}$ error.

\begin{tabular}{|c|c|c|c|c|}
\hline & Fr $=10$. & Fr $=1$. & Fr $=.1$ & Fr $=.01$ \\
\hline Upwind kinetic scheme & 0.427 & 0.906 & 1.099 & 1.125 \\
\hline Two time steps scheme & 0.427 & 0.412 & 0.192 & 0.110 \\
\hline
\end{tabular}

\subsection{Dam break}

We now consider the very classical case of a dam break on a flat bottom in which the concentration of pollutant is different on each side of the dam.

The interest of this second test is to present a more complex - and then more interesting - hydrodynamic background but preserving the existence of an exact solution for both hydrodynamic and pollutant problems.

The geometrical and time data are the following:

Spatial domain: $x \in[-1000,1000]$

Uniform mesh with 101 nodes.

Physical time: $240 \mathrm{~s}$.

We present three different cases by modifying only the water height on the right side of the dam. The hydrodynamic and pollutant initial data are - with the classical notations and with the subscripts 1 for the negative values of $\mathrm{x}$ and $\mathrm{r}$ for the positive ones

$$
\begin{aligned}
h_{l}=1 . & & h_{r}=\{.95 ; .8 ; .2\} \\
Q_{l}=0 . & & Q_{r}=0 . \\
T_{l}=.7 & & T_{r}=.5
\end{aligned}
$$

The analytic hydrodynamic solution of this problem is given in [19]. It is composed of three flat zones - the two original inactive zones at the two ends and an intermediate one - separated by two simple waves - a rarefaction wave going to the left and a shock wave going to the right. And the jump in the concentration is just transported at the speed $u_{i}$ of the intermediate flat zone which is given by the relation

$$
-2 u_{i}^{2}\left(c_{l}-\frac{u_{i}}{2}\right)^{2} c_{r}^{2}+\left(\left(c_{l}-\frac{u_{i}}{2}\right)^{2}-c_{r}^{2}\right)\left(\left(c_{l}-\frac{u_{i}}{2}\right)^{4}-c_{r}^{4}\right)=0
$$

where $c_{l}$ and $c_{r}$ are defined by

$$
c_{l}^{2}=g h_{l}, \quad c_{r}^{2}=g h_{r} .
$$

We present in Figure 3 the water height and the discharge profiles for the large jump $-h_{r}=.2$. It appears that the velocity of the shock and the water height of the intermediate flat zone are well captured.

Then as for the first test case we exhibit in Table 3 the auto-adaptive aspect of the method in terms of ratio between the number of hydrodynamic time steps and the number of transport time steps.

TABLE 3. Comparison between numbers of hydrodynamic and transport time steps.

\begin{tabular}{|c|c|c|c|c|}
\hline$h_{l}$ & $h_{r}$ & Froude number & Transport Steps & Hydrodynamic Steps \\
\hline 1. & .95 & 0.026 & 1 & 47 \\
\hline 1. & .8 & 0.112 & 5 & 48 \\
\hline 1. & .2 & 0.804 & 27 & 54 \\
\hline
\end{tabular}

Finally we present in Figure 4 the zoom view of the discontinuous area for the concentration of pollutant for the three cases. 

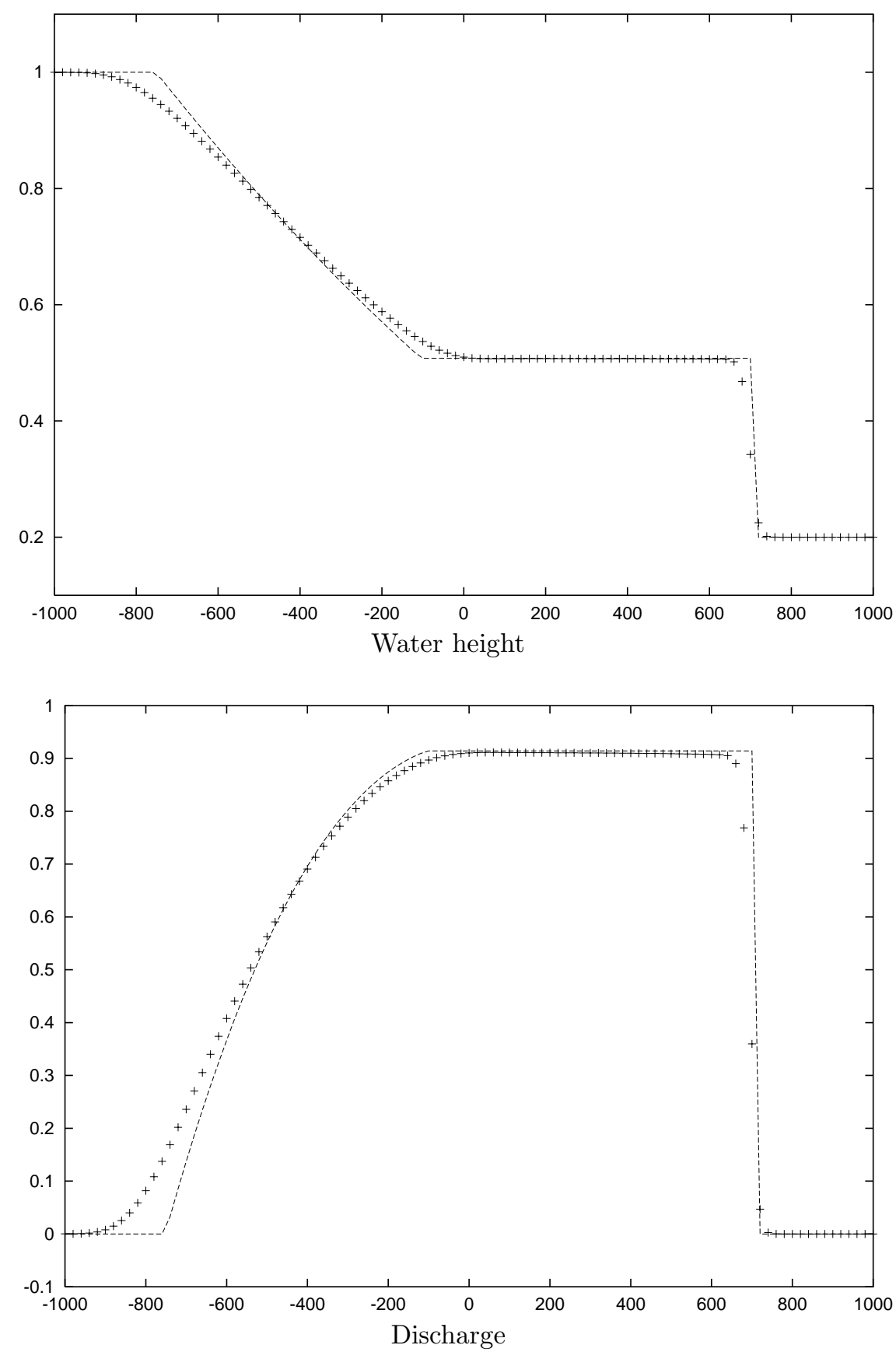

Figure 3. Dam break on a flat bottom: hydrodynamic results. Exact solution (dash line); kinetic scheme (crosses). 

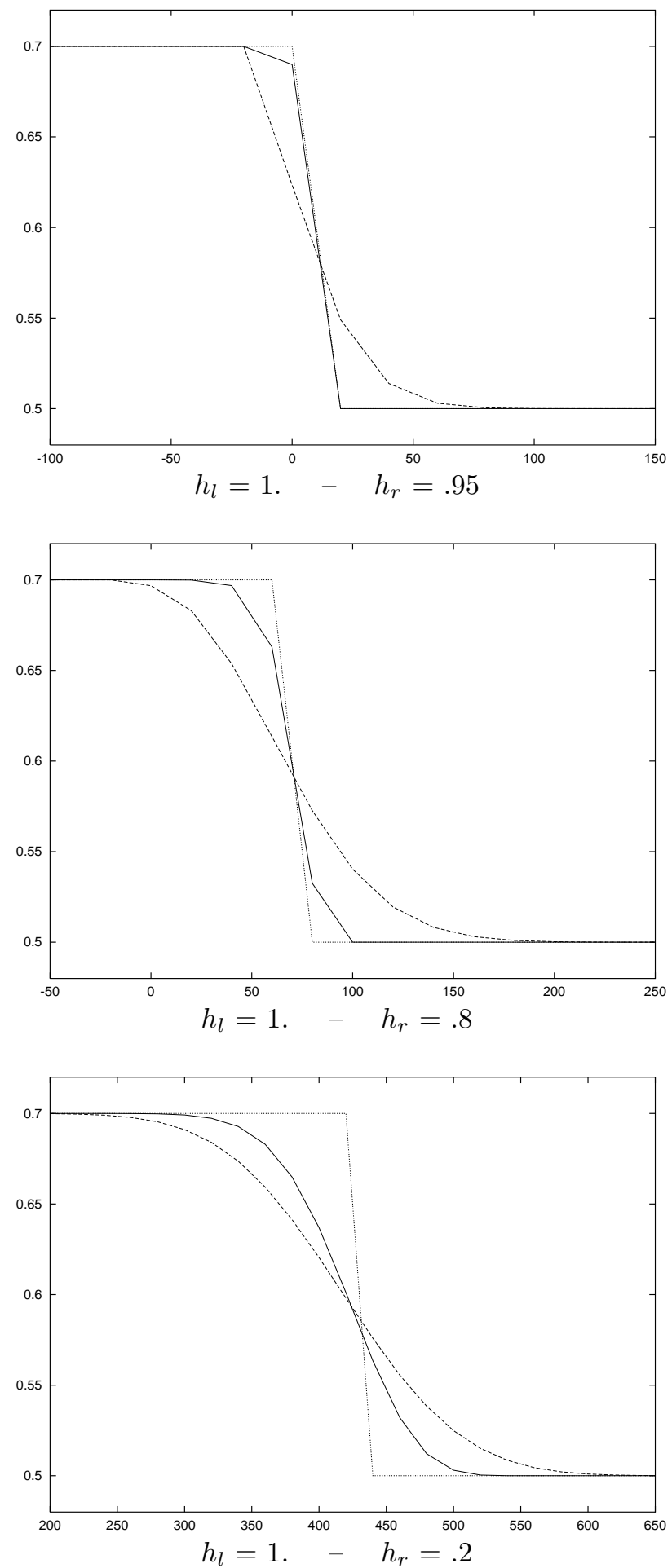

Figure 4. Concentration of pollutant for some dam break problems. Exact solution (dotted line); upwind scheme (dash line); two time steps scheme (continuous line). 
Let us notice some remarks: First the pollutant shock front is well captured by both schemes and its speed grows with the size of the water height jump as it is given by the formula (6.1). Second - as for the first test case - the two time steps solution is always better than the upwind one and the difference is more important when the jump is smaller - i.e. when the Froude number is smaller.

\subsection{Peak in the concentration of pollutant}

The hydrodynamic initialization is still a dam break. The concentration of pollutant is still different on each side of the dam. But now there is a peak in the concentration of pollutant just before the dam.

In this third test case we mix the first and the second one. The hydrodynamic background is quite complex and the initial concentration of pollutant present some oscillations. The $1 \mathrm{D}$ results presented in this section will be later compared with a $2 \mathrm{D}$ simulation.

The numerical values are the same as in the first example of the previous section excepted the initial value for the concentration of pollutant which is

$$
\begin{aligned}
-1000 . \leq x<-100 .: & T(x, 0)=T_{l}=0.7 \\
-100 . \leq x \leq 0 .: & T(x, 0)=T_{i}=0.9 \\
0 .<x \leq 1000 .: & T(x, 0)=T_{r}=0.5 .
\end{aligned}
$$

We do not have an analytical solution for this case. But we know the initial value at the node $x=0$. will be simply transported with the speed $u_{i}$ given by (6.1). So as we prove on an other hand a maximum principle for the concentration of pollutant we know its maximum value will stay equal to $T_{i}$.

On the first plot of the Figure 5 are presented the two numerical solutions after $250 \mathrm{~s}$. We indicate the initial solution too. As for the second case we observe a shock front and as for the first case the large diffusion of the upwind kinetic scheme conducts to loose the exact maximum value of the concentration of pollutant while the two time steps scheme computes it very precisely. Informations about the comparison between the numbers of time steps are given in a following section.

\subsection{Emission of pollutant in a non flat bottom channel}

Here we want to test the introduction of a source of pollutant in a stationary flow on a non flat bottom. It is the academic 1D version of a $2 \mathrm{D}$ problem that will be presented later and that models for instance the emission of waste water in a river.

We consider an academic parabolic bottom profile with a length of $500 \mathrm{~m}$

$$
Z(x)=\left(0.2-0.05(x-250)^{2}\right)_{+},
$$

and we use a uniform mesh with 101 points of discretization.

Then there exist four different hydrodynamic stationary cases following the different fluid states - fluvial or torrential flows. See [1] or [16] for more details. Here we choose to work with the fluvial flow problem where the improvement due to the two time steps scheme is very clear because of a small Froude number.

$$
\begin{aligned}
& h(x, 0)=H=2 . \\
& Q(x, 0)=Q=1 .
\end{aligned}
$$

We introduce between times $t_{b}$ and $t_{e}$ and at the node $I_{s}$ a source of water $S_{s}$ with a concentration of pollutant $T_{s}$. Then we follow the evolution of this pollutant layer. Notice that the source modifies locally in time and space the hydrodynamic computation.

$$
\begin{aligned}
& I_{s}=10, \quad S_{s}=.01 \mathrm{~m} \mathrm{~s}^{-1}, \quad T_{s}=10 \\
& t_{b}=100 \mathrm{~s}, \quad t_{e}=300 \mathrm{~s} .
\end{aligned}
$$



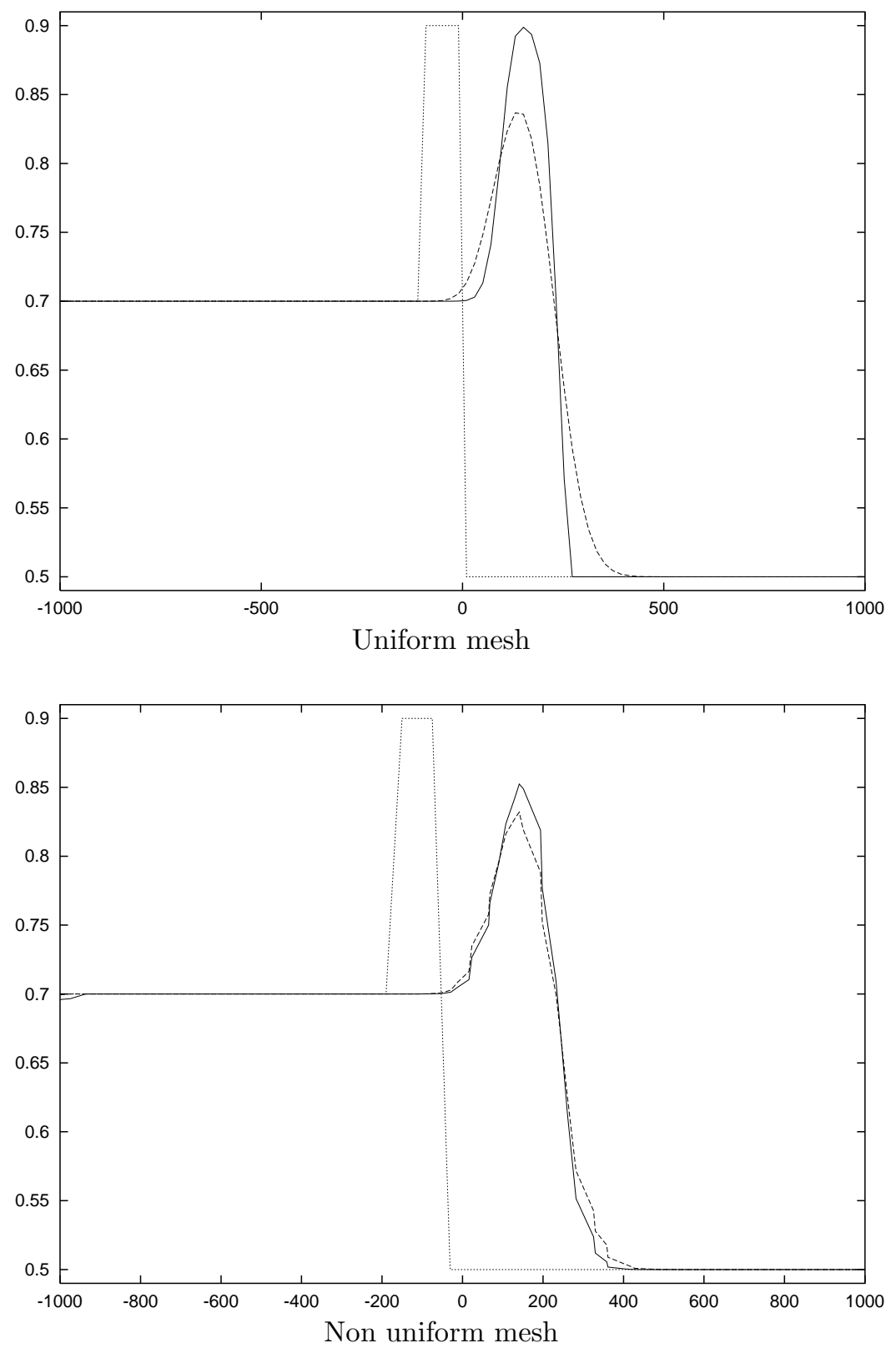

Figure 5. Concentration of pollutant for the peak problem. Initial data (dotted line); upwind scheme (dash line); two time steps scheme (continuous line).

Numerical results are presented in Figure 6 and informations about the number of time steps are given in the next section.

\subsection{With a non uniform mesh}

We mention before that the two time steps method allows an important improvement in term of accuracy. Here we want to exhibit - through some numerical examples - that this improvement is very related to the 

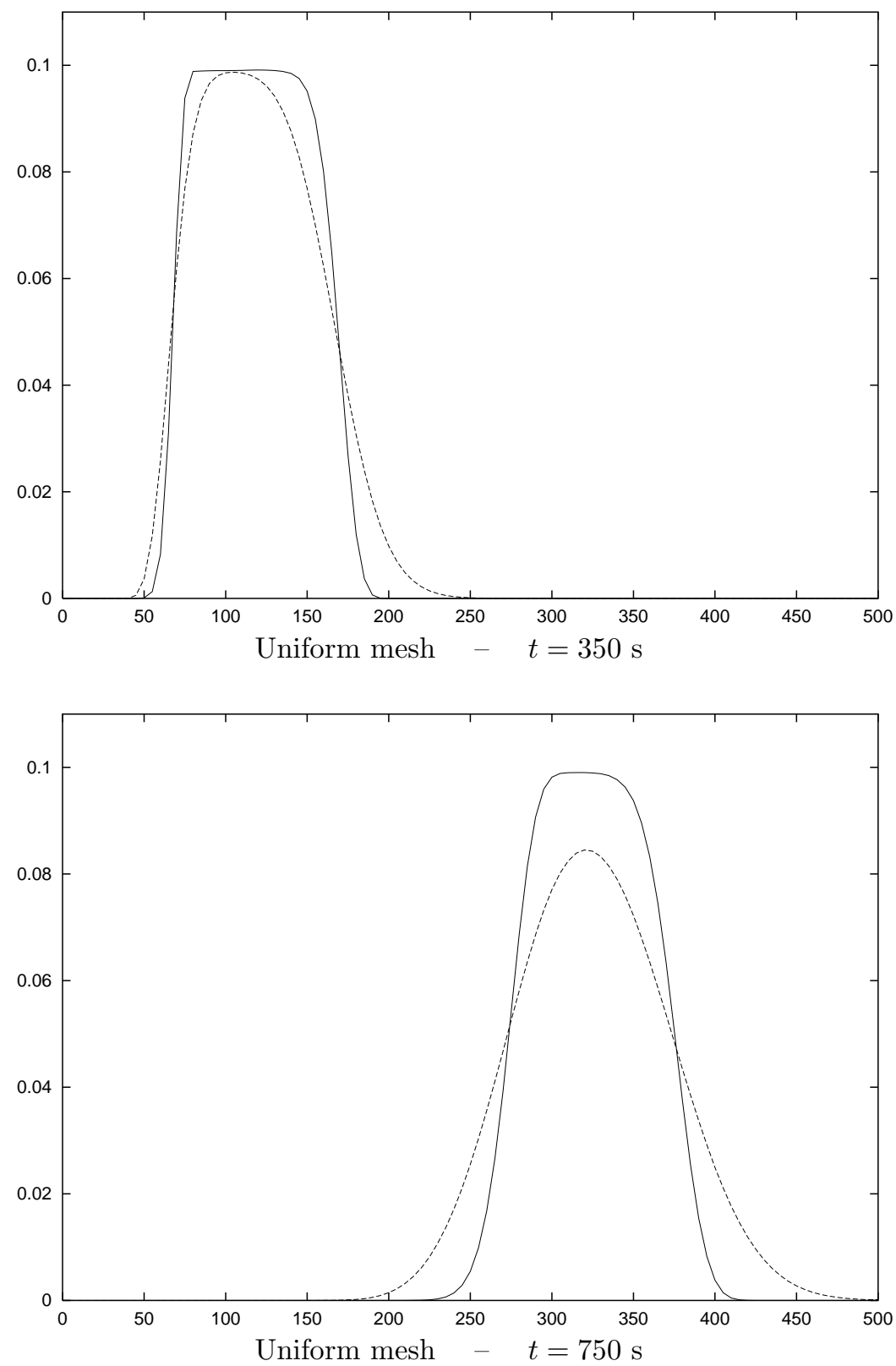

Figure 6. Concentration of pollutant - Emission of pollutant in a fluvial flow over a bump. Upwind scheme (dash line); two time steps scheme (continuous line).

regularity of the mesh. We introduce a non-uniform mesh - which is a controlled random perturbation of the uniform one - and we characterize it through its minimum and maximum space steps

$$
\begin{aligned}
& \Delta x_{\min }=0.33316 \\
& \Delta x_{\max }=1.81694 .
\end{aligned}
$$


Then we come back on the two last test cases. We begin with the dam break problem with a peak in the concentration of pollutant. We compute this test on the new mesh and with the two methods - the upwind kinetic scheme and the two time steps kinetic scheme - and we present the results on the second plot of the Figure 5. We first remark that more irregular is the mesh more the CFL condition is hard since it is related to the lower space step. So the number of time steps and then the numerical diffusion increase with the irregularity of the mesh and the precision of the results decreases. On an other hand it appears clearly that the difference in the precision of the results between the upwind and the two time steps methods decreases when the mesh is very irregular. However the two time steps method is always more accurate than the upwind one and - that is the essential point - we show in Table 4 that the ratio between the number of transport and hydrodynamic time steps is independent of the regularity of the mesh. So there is always an important improvement on the computation time and on the storage.

TABLE 4. Comparison between different meshes for a dam break problem with a peak in the concentration of pollutant.

\begin{tabular}{|c|c|c|}
\hline Mesh & Transport Steps & Hydrodynamic Steps \\
\hline Regular mesh & 13 & 53 \\
\hline Semi-random mesh & 30 & 148 \\
\hline
\end{tabular}

Then we compute the emission of pollutant problem of the precedent section. We apply the upwind and the two time steps methods on our new mesh and we present the results for two times $-350 \mathrm{~s}$ and $750 \mathrm{~s}-$ in the Figure 7. We present the informations about the number of time steps in Table 5. The conclusions are the same as for the dam break problem.

TABLE 5. Comparison between different meshes for an emission of pollutant problem.

\begin{tabular}{|c|c|c|c|}
\hline Mesh & Physical time & Transport Steps & Hydrodynamic Steps \\
\hline Regular mesh & 350 & 42 & 416 \\
\hline Regular mesh & 750 & 89 & 890 \\
\hline Semi-random mesh & 350 & 114 & 1246 \\
\hline Semi-random mesh & 750 & 243 & 2669 \\
\hline
\end{tabular}

\section{Extension to THE 2D CASE}

We now want to apply our new method to two-dimensional problems. We do not want to make here a complete presentation of the two dimensional finite volume method on a general triangular grid or of the general kinetic theory in 2D. We just mention that starting from a triangulation of $\mathbb{R}^{2}$ the dual cells $C_{i}$ are obtained by joining the centers of mass of the triangles surrounding each vertex $P_{i}$. Then the general method is close to the 1D finite volume method. Indeed the fluxes which appear in the scheme are interpolations of the normal component of the fluxes on the edge of each cell. So locally the problem can be treated as a planar discontinuity and the interpolation can be performed using a one dimensional solver. For a complete presentation of the kinetic interpretation of the hydrodynamic part in $2 \mathrm{D}$ refer to [1] and for a presentation that includes the transport theory see [4]. Before we present the numerical results let us insist on the fact that as the 2D computation is based on a 1D strategy the properties we proved in $1 \mathrm{D}$ are also true for the $2 \mathrm{D}$ schemes. We do not reproduce the proofs here because they are easy extensions of those in Section 4.

We perform two numerical tests: first a dam break problem in a rectangular channel with a peak in the initial concentration of pollutant - see the Section 6.2 in 1D - and then an emission of pollutant problem in a realistic river geometry. 

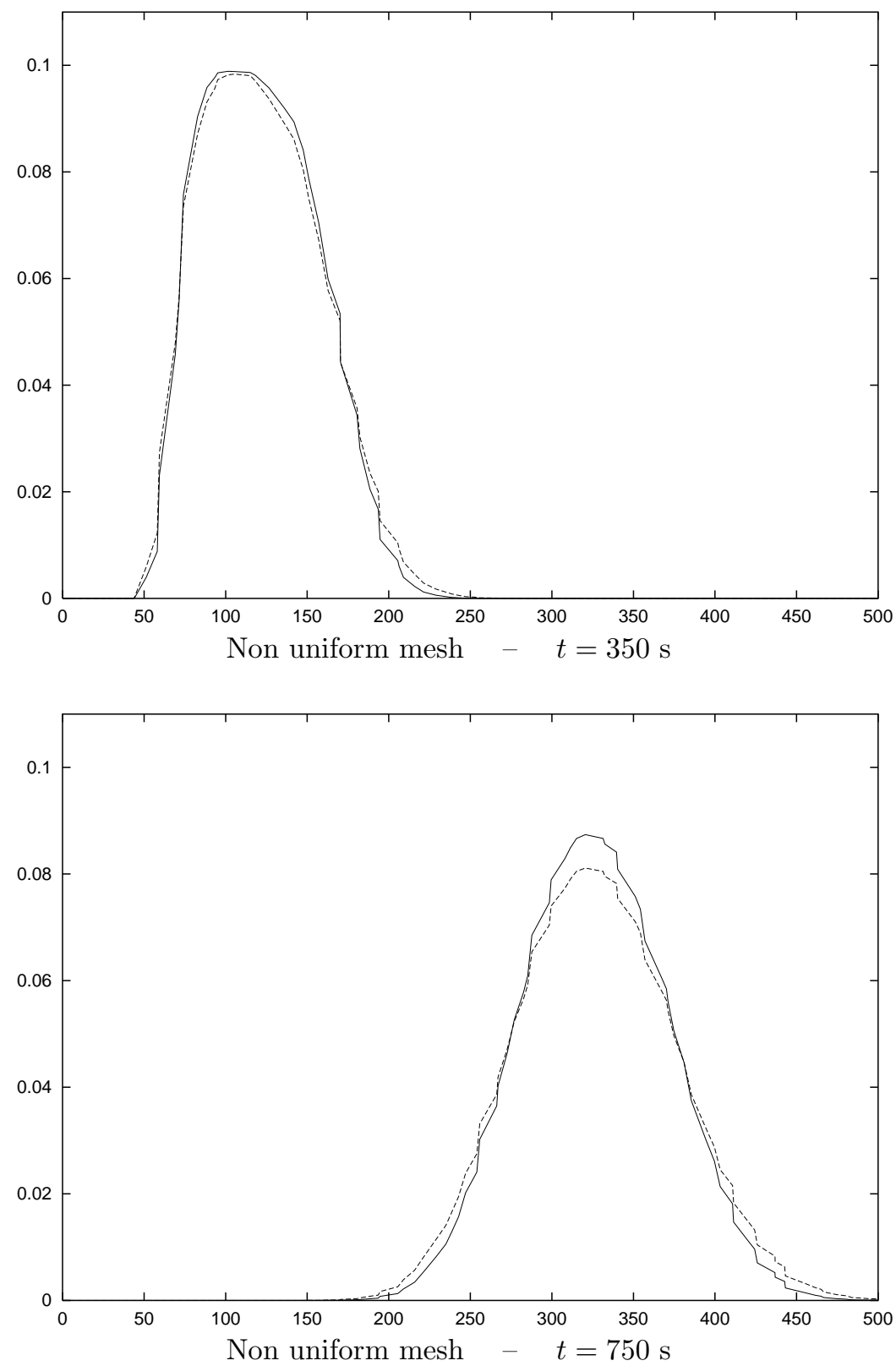

Figure 7. Concentration of pollutant - Emission of pollutant in a fluvial flow over a bump. Upwind scheme (dash line); two time steps scheme (continuous line).

For the dam break problem we use two meshes: a uniform one and an unstructured one. Then as in the 1D case we can compare the results based on the mesh regularity. The uniform mesh has 1111 nodes and 2000 elements and the unstructured one has 1347 nodes and 2472 elements. As the dam break case is essentially a $1 \mathrm{D}$ problem we can also make comparisons between 1D and 2D solutions. 


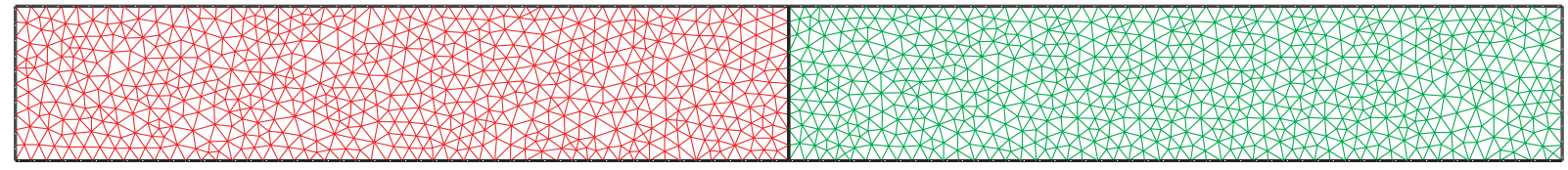

Unstructured mesh

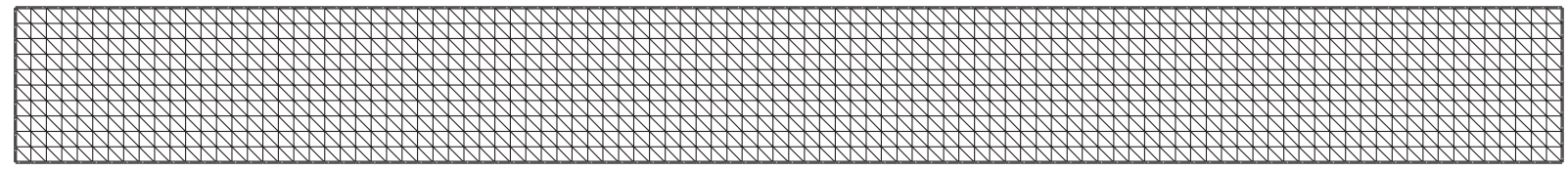

Uniform mesh

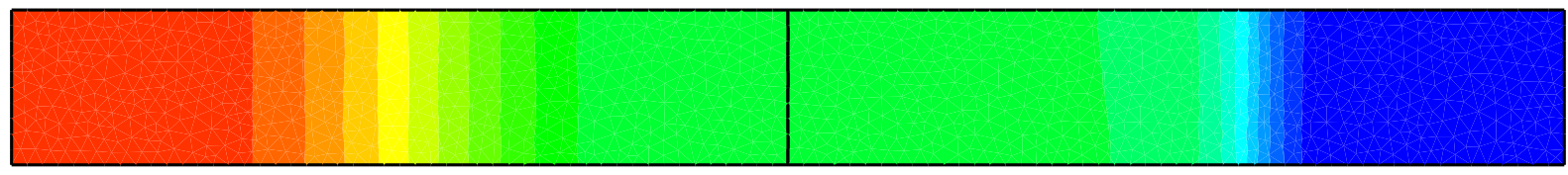

Water height

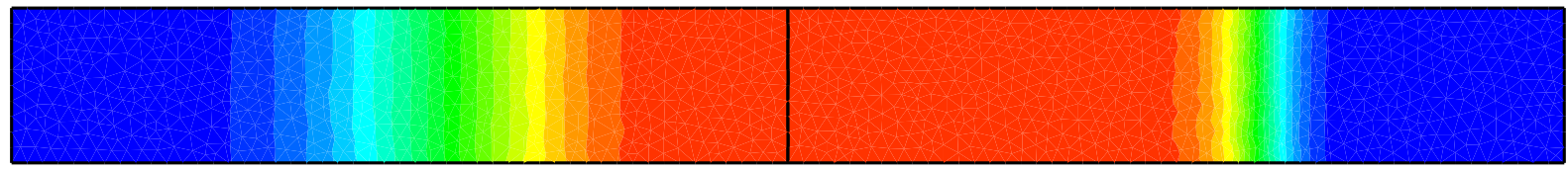

Froude Number

FiguRE 8. 2D dam break - Meshes and hydrodynamic results.

The realistic river case is managed only with an unstructured mesh and exhibits that our method is well adapted to treat complex geometry since the gain on the number of time steps is even more important than for the academic problems.

\subsection{A 2D dam break problem}

We begin with the dam break problem. The data are the same as for the problem performed in the Section 6.2 except the length of the channel which is now one meter - and so the final time is only one second. The width is chosen equal to $0.1 \mathrm{~m}$.

The results are presented in Figures 8 and 9. The data are invariant in $y$-coordinates and so the solution is very close to a $1 \mathrm{D}$ solution. For each figure the blue color indicates the minimum level and the red color the maximum one.

In Figure 8 we first present the two meshes. Then we present the water height in the channel. On the left we recognize the rarefaction wave and on the right the shock wave. Then on the last plot the Froude number is presented. The profile is very close to the discharge profile in $1 \mathrm{D}$.

In Figure 9 is first presented the initial data for the pollutant concentration and then on the four last plots are presented the concentration of pollutant performed with the upwind kinetic scheme and with the two time steps scheme on the unstructured mesh and then the same results with the uniform one. The profiles are very similar to the $1 \mathrm{D}$ profile. With the unstructured mesh the two profiles are very close - even if the maximum 


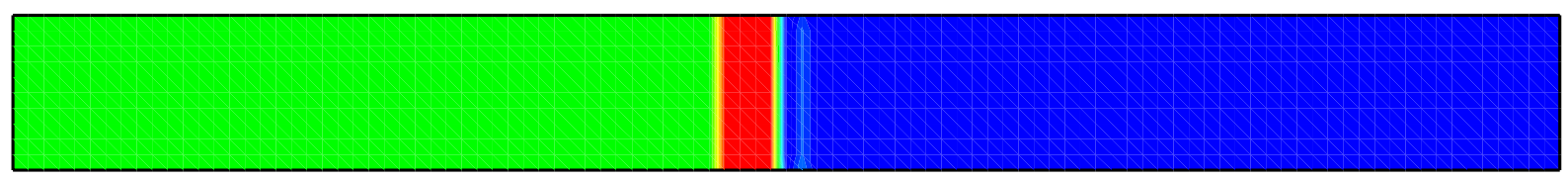

Initial solution

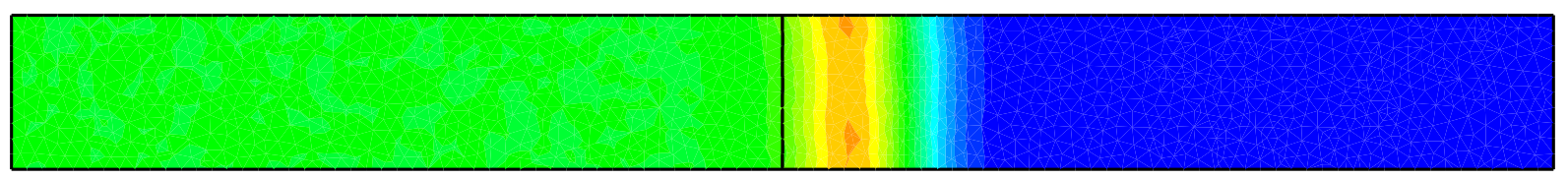

Unstructured mesh - Upwind kinetic scheme

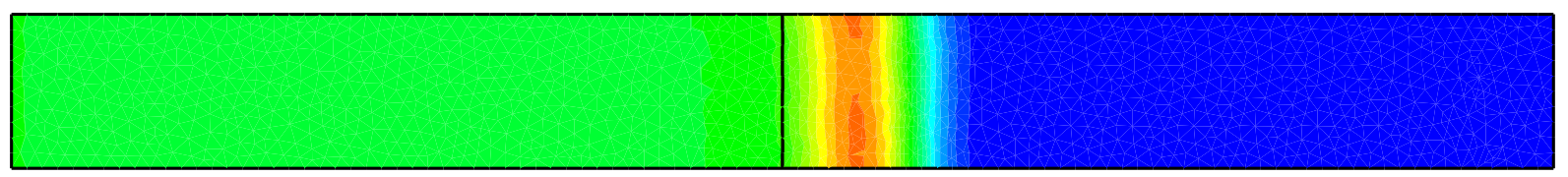

Unstructured mesh - Two time steps kinetic scheme

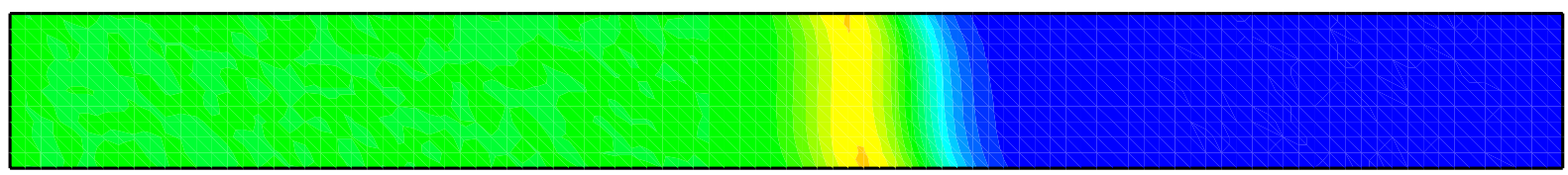

Uniform mesh - Upwind kinetic scheme

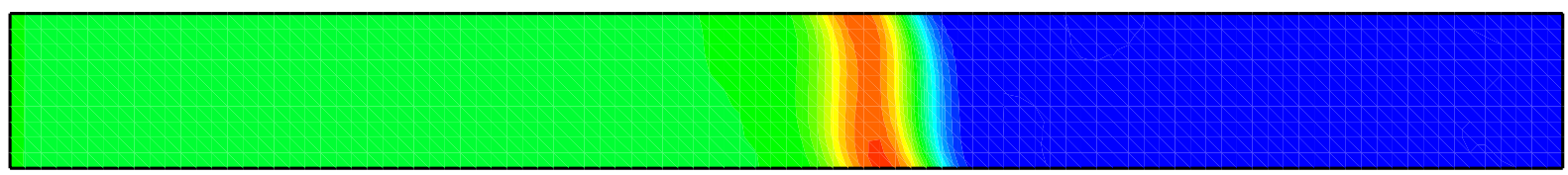

Uniform mesh - Two time steps kinetic scheme

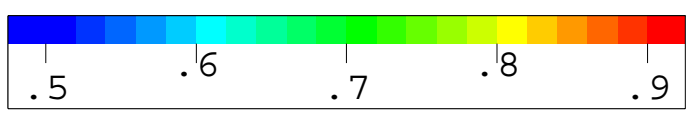

Scale for concentration of pollutant values

Figure 9. 2D dam break - Concentration of pollutant.

of the concentration is a little larger with the two time steps method as in the $1 \mathrm{D}$ computation when the mesh is non-uniform. With the uniform mesh the profiles are more different. With the upwind kinetic scheme the results are worse than on the unstructured mesh - notice that the uniform mesh has less nodes - but with the 

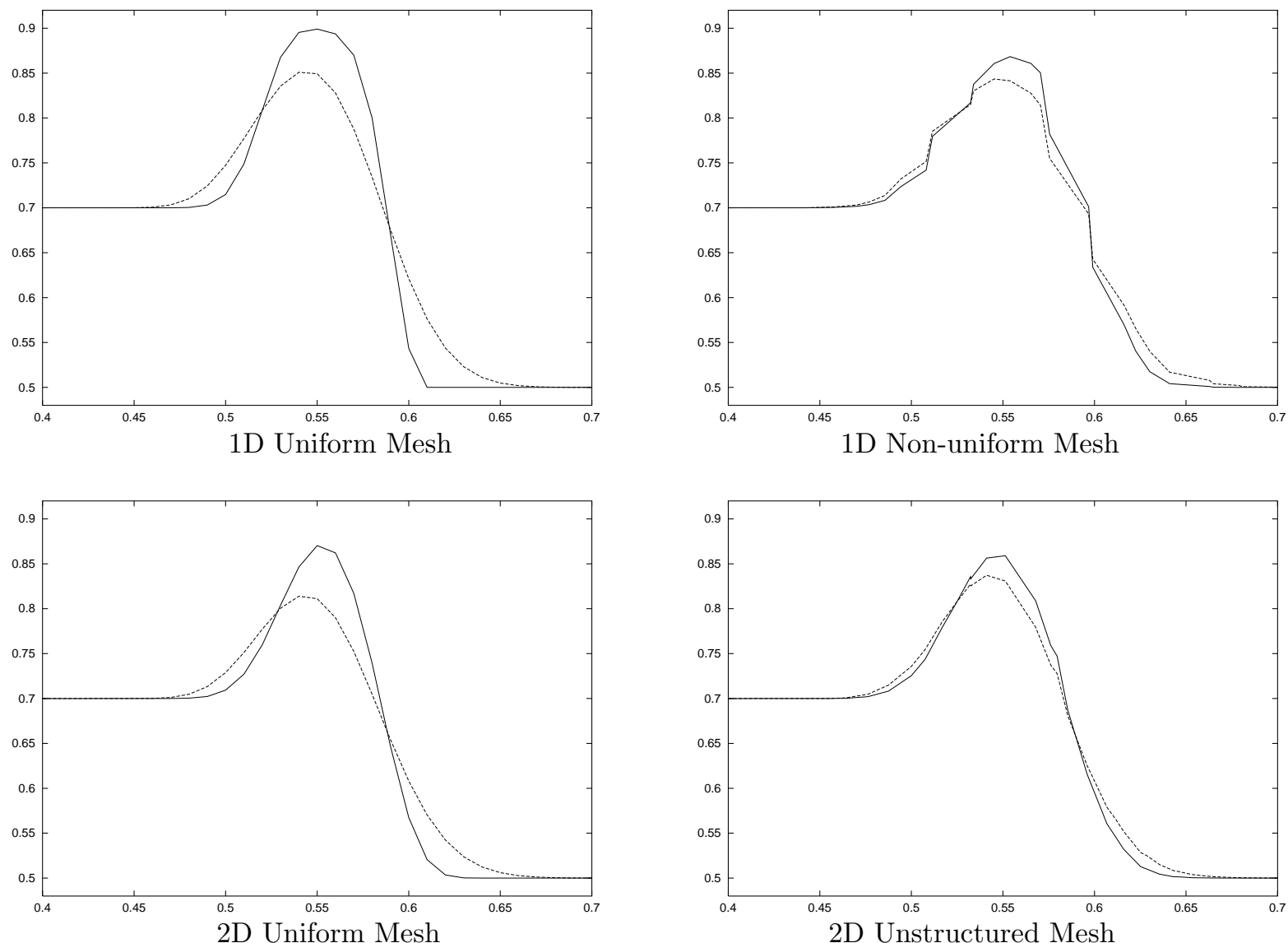

Figure 10. Concentration of pollutant for a peak problem. Comparison between the 1D problem and the $2 \mathrm{D}$ problem (plane $y=0.5$ ); upwind scheme (dash line); two time steps scheme (continuous line).

two time steps scheme the results are better than on the unstructured mesh. So as in the $1 \mathrm{D}$ computation the improvement on the accuracy of the results is more significant when the mesh is more regular.

Before to end with this problem we make a comparison between the $1 \mathrm{D}$ and the $2 \mathrm{D}$ results. So we perform the computations with the same initial conditions and we consider the $2 \mathrm{D}$ results on the line $y=0.5$. Then we present in Figure 10 the results on the two 1D meshes and on the two 2D meshes. We can see that the regularity of the mesh has the same effects in $1 \mathrm{D}$ and in $2 \mathrm{D}$ - even if the $1 \mathrm{D}$ uniform mesh induces a smaller diffusion than the 2D uniform mesh.

\subsection{Emission of pollutant in a realistic river}

Then we perform an emission of pollutant problem in a river. The geometric data include a jetty in the transversal direction and a bridge pillar. We introduce a source of pollutant at one node of the mesh and for a given time - from $1000 \mathrm{~s}$ to $1800 \mathrm{~s}$ - and we follow the evolution of the pollutant layer.

We use a second order scheme to compute the hydrodynamic part because the first order solution does not show the recirculation after the jetty which is very important to compute a realistic profile of the concentration of pollutant. We keep a first order solution for the transport part not to mix the improvement due to high order schemes and those due to the applied method. Results are presented in Figures 11 and 12 . Here the mesh is 


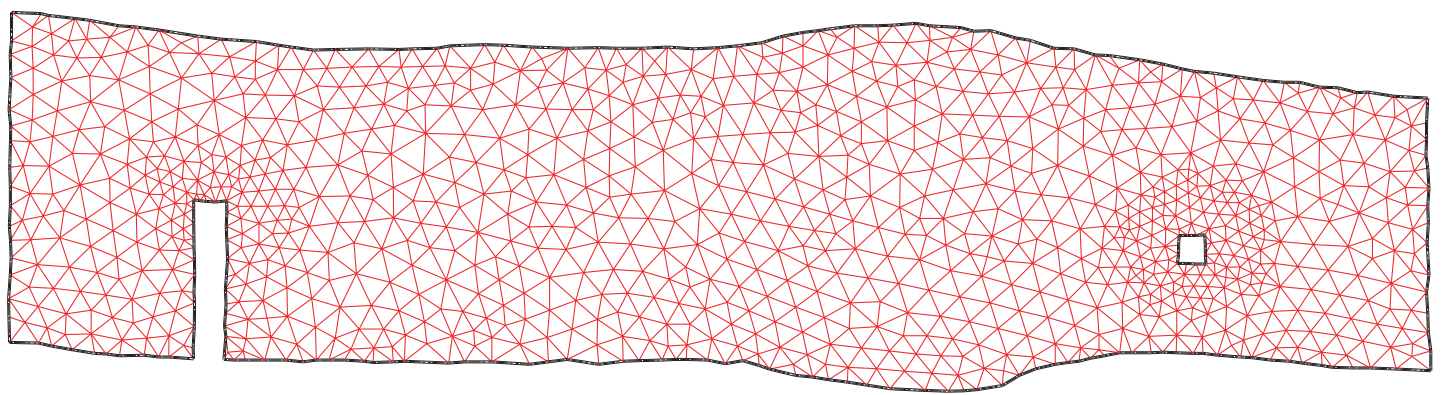

The mesh

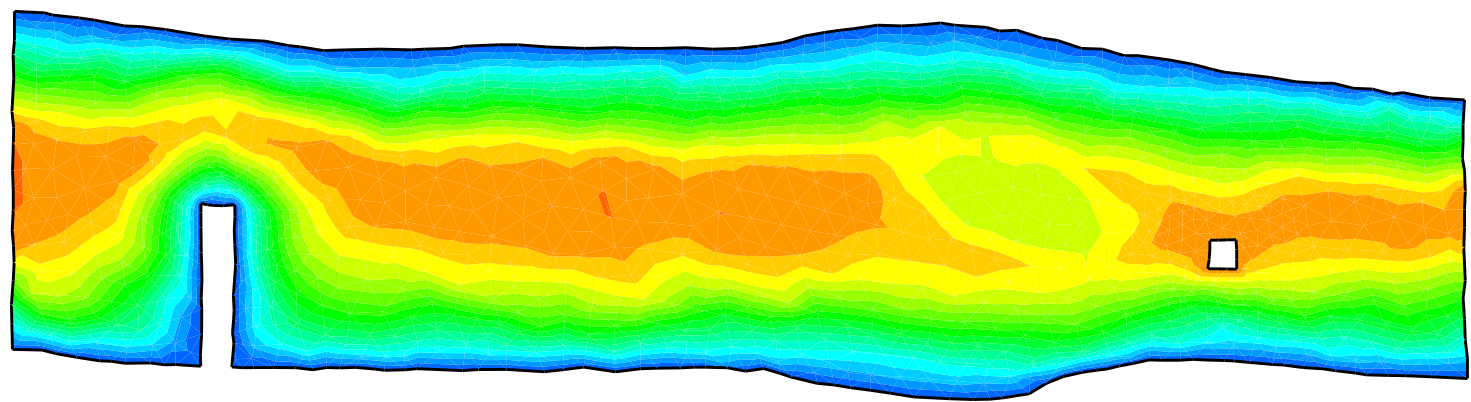

Water height

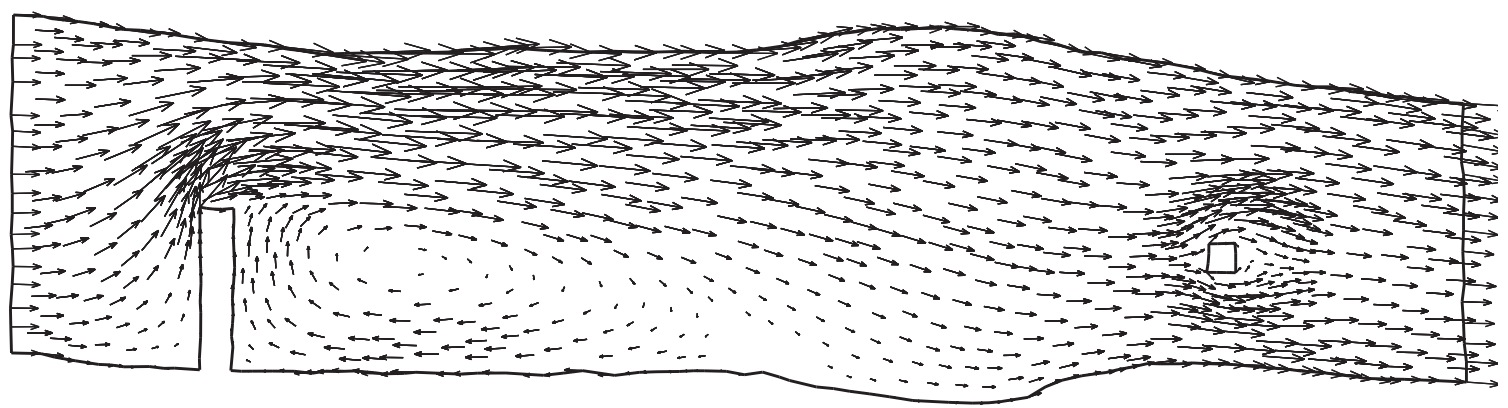

Velocity

FIgURE 11. River with emission of pollutant - Mesh and hydrodynamic results.

unstructured and so the concentration of pollutant profiles are very close. But as in the other cases the two time steps scheme is a little bit better.

\section{Conclusion}

Thanks to a precise analysis of the upwind kinetic scheme we have deduced a two time steps kinetic scheme that preserves the theoretical properties of the upwind kinetic scheme. By opposition to the hydrodynamic CFL condition, the new transport time step condition links automatically the transport time step, the space step and the fluid velocity, ignoring the sound speed. 


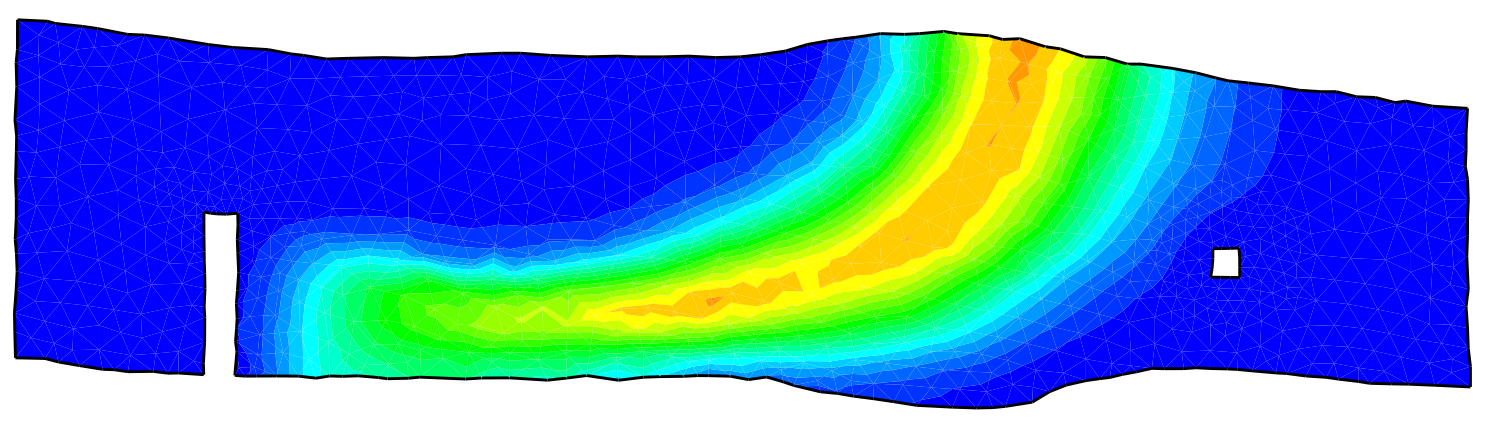

Concentration of pollutant - Upwind kinetic scheme

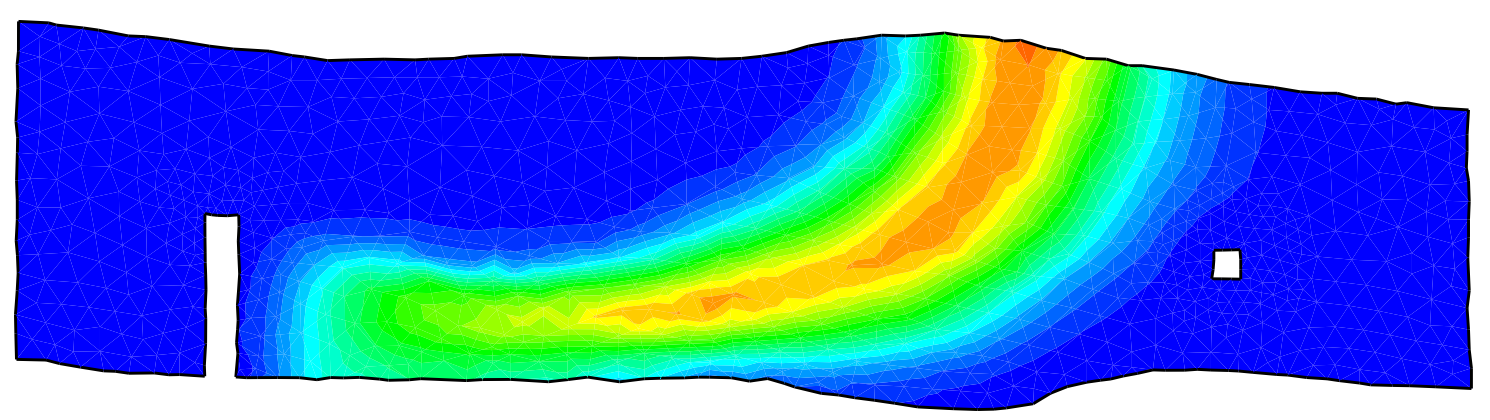

Concentration of pollutant - Two time steps kinetic scheme

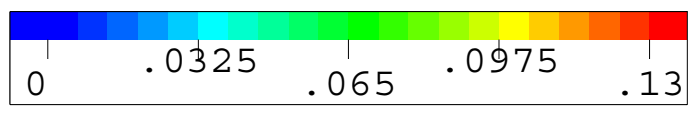

Scale for concentration of pollutant values

FiguRE 12. River with emission of pollutant - Concentration of pollutant.

The hydrodynamic part of the computation remains unchanged. The whole hydrodynamic information that is useful for the pollutant transport computation can be stored in global interface fluxes at each transport time step.

The developed method is very interesting for the small Froude number flows. Indeed the two time steps are very close when the speed of the flow is large compared with the sound speed but they are very different in the other case. As we can see in Table 6, in a 2D realistic geometry and for a Froude number close to 0.1 - which is a usual order of magnitude for rivers, the new transport time step is around hundred and fifty times larger than the hydrodynamic time step issued from the CFL condition.

TABLE 6. Comparison between numbers of hydrodynamic and transport time steps for the 2D test cases.

\begin{tabular}{|c|c|c|c|}
\hline & Froude number & Transport Steps & Hydrodynamic Steps \\
\hline Unstructured channel & 0.35 & 28 & 385 \\
\hline Uniform channel & 0.37 & 14 & 170 \\
\hline Realistic river & 0.08 & 320 & 45637 \\
\hline
\end{tabular}


The improvement is proved to be optimal if we want to preserve a priori the non-negativity properties of the upwind kinetic scheme.

As the new time step condition is specifically adapted to the transport equation the numerical diffusion of the two time steps kinetic scheme is lower than the numerical diffusion of the upwind kinetic scheme and we therefore improve the accuracy of the results.

Acknowledgements. This research has been partially supported by EDF/LNHE. The authors thank Benoit Perthame and Jean-Michel Hervouet for helpful discussions.

\section{REFERENCES}

[1] E. Audusse, M.O. Bristeau and B. Perthame, Kinetic schemes for Saint-Venant equations with source terms on unstructured grids. INRIA Report, RR-3989 (2000), http://www.inria.fr/RRRT/RR-3989.html.

[2] A. Bermudez and M.E. Vasquez, Upwind methods for hyperbolic conservation laws with source terms. Comput. \& Fluids 23 (1994) 1049-1071.

[3] M.O. Bristeau and B. Coussin, Boundary conditions for the shallow water equations solved by kinetic schemes. INRIA Report, RR-4282 (2001), http://www.inria.fr/RRRT/RR-4282.html.

[4] M.O. Bristeau and B. Perthame, Transport of pollutant in shallow water using kinetic schemes. CEMRACS, ESAIM Proc. 10 (1999) 9-21, http://www.emath.fr/Maths/Proc/Vol.10.

[5] R. Eymard, T. Gallouet and R. Herbin, Finite volume methods, Handbook of numerical analysis, Vol. VIII, P.G. Ciarlet and J.L. Lions Eds., Amsterdam, North-Holland (2000).

[6] T. Gallouet, J.M. Hérard and N. Seguin, Some approximate Godunov schemes to compute shallow water equations with topography. Comput. \& Fluids 32 (2003) 479-513.

[7] J.F. Gerbeau and B. Perthame, Derivation of viscous Saint-Venant system for laminar shallow water; Numerical validation. Discrete Contin. Dynam. Systems 1 (2001) 89-102.

[8] E. Godlewski and P.A. Raviart, Numerical approximation of hyperbolic systems of conservation laws. Springer-Verlag, New York, Appl. Math. Sci. 118 (1996).

[9] L. Gosse and A.Y. LeRoux, A well-balanced scheme designed for inhomogeneous scalar conservation laws. C. R. Acad. Sci. Paris Sér. I Math. 323 (1996) 543-546.

[10] J.M. Hervouet, Hydrodynamique des écoulements à surface libre, apport de la méthode des éléments finis. EDF (2001).

[11] S. Jin, A steady state capturing method for hyperbolic system with geometrical source terms. ESAIM: M2AN 35 (2001) 631-646.

[12] R.J. LeVêque, Numerical Methods for Conservation Laws. Second edition, ETH Zurich, Birkhauser, Lectures in Mathematics (1992).

[13] R.J. LeVêque, Balancing source terms and flux gradients in high-resolution Godunov methods: the quasi-steady wavepropagation algorithm. J. Comput. Phys. 146 (1998) 346-365.

[14] L. Martin, Fonctionnement écologique de la Seine à l'aval de la station d'épuration d'Achères: données expérimentales et modélisation bidimensionnelle. Ph.D. Thesis, École des Mines de Paris, France (2001).

[15] B. Perthame, Kinetic formulations of conservation laws. Oxford University Press (2002).

[16] B. Perthame and C. Simeoni, A kinetic scheme for the Saint-Venant system with a source term. Calcolo 38 (2001) $201-231$.

[17] P.L. Roe, Upwind differencing schemes for hyperbolic conservation laws with source terms, in Nonlinear Hyperbolic Problems, C. Carasso, P.A. Raviart and D. Serre Eds., Berlin, Springer-Verlag, Lecture Notes in Math. 1270 (1987) 41-51.

[18] A.J.C. de Saint-Venant, Théorie du mouvement non permanent des eaux, avec application aux crues de rivières et à l'introduction des marées dans leur lit. C. R. Acad. Sci. Paris Sér. I Math. 73 (1871) 147-154.

[19] J.J. Stoker, The formation of breakers and bores. Comput. Appl. Math. 1 (1948). 